American Journal of Pharmaceutical Education 2018; 82 (5) Article 7160.

\title{
SCHOOL POSTERS
}

\section{9th Annual Meeting of the American Association of Colleges of Pharmacy, Boston, Massachusetts, July 21-25, 2018}

\begin{abstract}
A Cultural Competency Course in an Interprofessional Education (IPE) Setting. Henry Cohen, Touro College of Pharmacy-New York, David A. Forstein, Touro College of Osteopathic Medicine, Tipsuda Junsanto-Bahri, Touro College of Osteopathic Medicine, Jeffrey Gardere, Touro College of Osteopathic Medicine, Joyce Addo-Atuah, Touro College of Pharmacy-New York. The school poster describes the development and implementation of an IPE course in Cultural Competency. "Introduction to Cultural Competency" was introduced in an IPE setting in spring 2017 enrolling DO1 students of the Touro College of Osteopathic Medicine (TouroCom) and P1 students of Touro College of Pharmacy (TCOP) both of the Touro Harlem campus in New York to learn about, from, and with each other. The large class size (243 in 2017 \& 181 in 2018) was organized in 2 sessions ( $\mathrm{A}$ and $\mathrm{B}$ ) each session consisting of half of the constituent classes. The course objectives reflect both the accreditation bodies' IPE and Cultural Competency Standards. The course is directed and taught jointly by faculty from both schools as well as guest lecturers representing different population groups. Assessment consists of attendance (30\%), in-class IPE team (5 DO1 \& 3 P1) exercises (10\%), and a 2-part IPE student teams project (60\%); a health promotional campaign based on the Health Belief Model targeting their assigned population groups. Students' feedback through anonymous course-related surveys informs ongoing course improvement toward better learning and experiential outcomes. The in-class IPE sessions enabled students to explore diversity in the classroom to prepare them for the diverse patient populations they will serve and also to incorporate ethical principles and cultural competence into healthcare. Students are increasingly engaged in the course and the growing positive interaction and collaboration between both faculty and students of the 2 schools is tangible and worthy of emulation by other schools.
\end{abstract}

A Faculty Exchange Program to Strengthen Collaborations in the Classroom. Lisa Lebovitz, University of Maryland, Steven Fletcher, University of Maryland, Lauren M. Hynicka, University of Maryland. A P1 Fall course at the University of Maryland School of Pharmacy, entitled General Patient Management (GPM), was purposefully designed by faculty members Dr. Hynicka, a pharmacist, and Dr. Fletcher, a basic scientist, to introduce new pharmacy students to the clinical thought process of patient diagnosis and treatment. GPM was constructed to provide a general approach to the collection and assessment of patient subjective and objective data, built on a firm foundation of clinical chemistry, using the Pharmacists' Patient Care Process (PPCP). To familiarize themselves with their different perspectives and approaches to the subject matter, the co-course managers spent time in each other's laboratory or clinical practice site. Shadowing Dr. Fletcher in the lab reinforced Dr. Hynicka's respect of the role of basic science in pharmacy, and re-energized her passion for chemistry. Rounding with Dr. Hynicka gave Dr. Fletcher insight into the applicability of medicinal chemistry concepts to pharmacy practice, leading him to tailor his teaching to those relevant principles. Both experiences deepened their mutual appreciation and helped them to mold the course into one that successfully bridges the basic sciences with pharmacy practice.
An Integrated Course Series: Applying the Pharmacists' Patient Care Process to Achieve Common Learning Outcomes. Kathleen Frey, Long Island University, Timothy V. Nguyen, Long Island University, Elaine Wong, Long Island University. The implementation of the ACPE Standards 2016 created an opportunity for the LIU Pharmacy PharmD program to undergo a curricular reform. A nine-course series entitled Modular Organ Systems Therapeutics Sequence or "MOST" was designed to integrate medicinal chemistry, pharmacology and therapeutics courses beginning in the P2 year of Fall 2016. Each MOST was taught in a block format with variable credit and time allocation depending on the organ system. The MOST series was linked with learning objectives, learning outcomes (LOs), and educational and assessment activities. Common LOs within each MOST included: learner, problem-solver, and caregiver. To achieve these LOs, a minimum of one integrated workshop was incorporated into each MOST. The workshop aligned with the Pharmacists' Patient Care Process utilizing a comprehensive patient case to bridge basic and clinical sciences. A tool was developed to guide students through expectations of the activity to identify and formulate responses for the following areas: collect (findings), assess (risk factors; medication appropriateness, effectiveness, safety, and adherence; preventative care; relevant drug mechanism of actions and structure activity relationships; plan and implementation (goals; therapy; education and self-management); follow-up (efficacy, safety). Dependent on the topic, additional student assessment activities were implemented pre- or post- workshop. Furthermore, the workshop and respective tool assisted with standardizing approaches to assessing LOs and establishing uniform expectations for both faculty and students within an integrated course. After a complete iteration, the integrated course will be evaluated for its impact on student learning, retention and application of medicinal chemistry, pharmacology and therapeutics.

An Interactive and Integrated Pharmacotherapy and Skills-Based Curriculum: Bridging Basic and Clinical Science. Joseph A. Barone, Rutgers, The State University of New Jersey, Carol Goldin, Rutgers, The State University of New Jersey, Lauren Aleksunes, Rutgers, The State University of New Jersey, Evelyn R. HermesDeSantis, Rutgers, The State University of New Jersey. The Ernest Mario School of Pharmacy launched a review and revision of its PharmD curriculum in 2016, using a systematic approach with three stages: Design, Develop, Execute. During Design, working groups of basic and clinical science faculty reviewed curricula, assessed preparedness for Advanced Pharmacy Practice Experiences, and considered a variety of integration approaches. Emerging curricular initiatives included: 1) integration of pharmacology and pharmacotherapy courses into organ-defined modules across P2 and P3 years and 2) creation of a novel skills-based integrated Pharmacotherapy Assessment Skills Series (iPASS) reinforcing lecture content. Critical for iPASS was an expansive infrastructure upgrade with state-of-theart simulation suites for transitions of care, sterile admixture preparation, and patient simulation. During Develop, basic and clinical science faculty worked with university instructional designers to construct learner-centered courses addressing Tier 1 and 2 diseases from the 2016 ACCP Pharmacotherapy Curriculum Toolkit. In total, the new courses represent a re-alignment of $40 \%$ of the PharmD 


\section{American Journal of Pharmaceutical Education 2018; 82 (5) Article 7160.}

curriculum. Basic and clinical science faculty participated in workshops designed by university instructional designers and pharmacy faculty to create active learning strategies for $200+$-person classes. Evaluation of the first 4-hour workshop indicated high faculty satisfaction (mean 4.4 on a $0-5$ Likert scale) with enthusiasm for active learning approaches including: 1) student-generated questions; 2) lecture check; 3) think, pair, share; and 4) classroom games. Execute started in spring 2018 with the first iPASS course and continues in earnest for P2 students in fall 2018 with integrated coursework and assessments of basic science and clinical knowledge in the pharmacotherapy curriculum.

Assessing the Co-Curriculum across the Pharmaceutical Sciences and Pharmacy Practice Courses. JoLaine R. Draugalis, The University of Oklahoma, Michael Ihnat, The University of Oklahoma, Vincent C. Dennis, The University of Oklahoma, Melissa S. Medina, The University of Oklahoma. The University of Oklahoma College of Pharmacy created a plan to assess students' completion of and knowledge gained from activities in five co-curricular areas using a course management system (D2L). First, the Assessment Committee categorized all co-curricular activities into five umbrella areas: patient education, health fairs, and clinics; leadership; fitness/mental health; patient education; community service; and professional development. Then the committee tagged each of the five areas to the CAPE/ACPE Standards 3 and 4 as primary and secondary outcomes. The committee then outlined minimum requirements for each of the five areas over four years and included a menu of available co-curricular activity options for students to select from to fulfill the requirements. Finally, the committee linked the five co-curriculum areas to specific pharmaceutical, pharmacy practice/clinical, and social and administrative sciences courses across the didactic curriculum (years P1-P3). Each course is responsible for assessing its' co-curricular area using multiple-choice test questions that are aligned with the CAPE Domains 3 and 4 objectives and for assigning a percent of the course grade to the co-curricular assessment. Connecting a co-curricular area and activities with a specific course in the curriculum involves all faculty in the pharmaceutical, clinical, and social and administrative sciences, in the assessment of co-curricular outcomes. This allows all faculty to have involvement in and a common assessment mission for the co-curriculum.

A Synergistic Combination: Pharmaceutical, Social and Administrative Sciences in the Case Studies in Pharmacotherapy Courses. Erenie Guirguis, Palm Beach Atlantic University, Jonathan Jackson, Palm Beach Atlantic University, Mariette Sourial, Palm Beach Atlantic University, Mark F. Bonfiglio, Palm Beach Atlantic University, Adwoa O. Nornoo, Palm Beach Atlantic University. Objectives: The incorporation of the pharmaceutical sciences into courses emphasizing the Pharmacist Patient Care Process is accomplished in the Case Studies in Pharmacotherapy series. The current emphasis is to proactively include concepts in the pharmaceutical, social and administrative sciences in course assessments and piloting methods for a deeper integration into the case writing process. Methods: Beginning in Fall of 2017, students were required to be prepared to discuss the relationship between the pharmacology of each drug in the plan, specifically as it relates to modifying the symptoms or progression of the disease. This is reflected in the verbal defense in order to emphasize this critical link between pathophysiology and pharmacology. In the Spring of 2018, a formal step in the case writing process is being piloted as new cases are developed. The appropriate state of case development to solicit input has been a subject of exploration. Recent experience has indicated that prompt specific and useful commentary is obtained when input is solicited early in the development of the case. Results: Performance data are being tabulated. A focus group will be conducted at the end of the Spring 2018. This process will continue to be refined for subsequent offerings the courses. Contributions will be documented and shared with faculty for further improvement. Conclusions: Integrating pharmaceutical, social and administrative sciences through the course series is an effective way to utilize previous knowledge and develop the skills necessary to practice optimal pharmaceutical care.

Beyond the Script - Creating a Collaborative Culture for Student Engagement. Frank Romanelli, University of Kentucky, Jeff J. Cain, University of Kentucky, R. Kiplin Guy, University of Kentucky, Holly S. Divine, University of Kentucky, David Feola, University of Kentucky, Melody H. Ryan, University of Kentucky, Esther P. Black, University of Kentucky, Wendy Ramey, UK Healthcare, Clark Kebodeaux, University of Kentucky, Vincent J. Venditto, University of Kentucky. The curriculum at the University of Kentucky College of Pharmacy has been re-envisioned to harness the strengths of our faculty, UK Healthcare, and the changing landscape of pharmacy practice in the Commonwealth of Kentucky, the United States, and globally. Courses are coordinated by faculty from both Pharmacy Practice (PP) and Pharmaceutical Sciences (PS) with additional insight from pharmacy practitioners at UK Healthcare in order to facilitate collaboration between disciplines. Integration of content delivery along the continuum of basic science, clinical implementation, and patient outcomes provides an opportunity for students to make concrete connections to the physiology, pathophysiology, medicinal chemistry, pharmacology and pharmacotherapeutics. This paradigm serves as the platform for student-centered inquiry-based learning necessary to establish future leaders in pharmacy. Key metrics for success will be obtained in 2020 as the initial cohort completes the curriculum. One metric of success of the integrated curriculum is highlighted by the evaluation of dual-degree student enrollment. Prior to curricular change, enrollment in the PharmD, Masters of Pharmaceutical Sciences (MS/PS) dual-degree program averaged $4 \%$ per class. After curricular restructuring, enrollment increased to $9 \%$ with the class of 2020 and to $17 \%$ for the class of 2021 . The clear demarcation in student interest in the MS/PS program underscores the impact of an integrated curriculum on student engagement. Course-specific feedback and preceptor evaluations from Introductory Pharmacy Practice Experiences provide additional insight into student success. Early evidence suggests that the integrated curriculum has enabled our students to engage in active learning, bridge disciplines, and think beyond the script.

Bridging Pharmaceutical Sciences and Pharmacy Practice through a Required, Longitudinal CAPstone Research Experience. Jose O. Rivera, The University of Texas at El Paso, Sweta Andrews, The University of Texas at El Paso, Fatima Z. Alshbool, The University of Texas at El Paso, Jeri J. Sias, The University of Texas at El Paso, Fadi T. Khasawneh, The University of Texas at El Paso, Amanda M. Loya, The University of Texas at El Paso, Jessica M. ShenbergerTrujillo, The University of Texas at El Paso, Jennifer Hartman, The University of Texas at El Paso, Ian A. Mendez, The University of Texas at El Paso, Emily J. Christenberry, The University of Texas at El Paso, Margie E. Padilla, The University of Texas at El Paso. Innovation: The CAPstone experience is introduced by Pharmaceutical Sciences and Pharmacy Practice in the Professionalism, Innovation, Leadership, and Life Skills (PILLS) Course during the Spring of the first year. Each student is assigned to watch videos that highlight faculty research in a flipped classroom format. After review of videos, classroom time is utilized to debrief students and allow them to ask questions about the CAPstone experience. Students are then given time to reflect and rank researchers based on interests. Research projects and faculty mentors will be grouped into 4 pillars of scholarship: Pharmaceutical Sciences, Clinical Practice, Public 


\section{American Journal of Pharmaceutical Education 2018; 82 (5) Article 7160.}

Health and Social Sciences, and Teaching and Learning. Design: The CAPstone sequence is a required 4-semester, 1-credit hour course taken during the second and third professional years of the curriculum. The CAPstone sequence is overseen by an AIM (Aligned, Integrated, and Meaningful) coordinator to ensure appropriate progression throughout the 4 semesters. The course coordinators for each semester, representing integration between the pharmaceutical sciences and pharmacy practice, are responsible for providing training and instruction on the basic components of the research method. The first semester will focus on research ethics training, literature review, background, and objectives. The second semester will focus on abstract, methods, and research board approvals. The third semester will focus on data collection/analysis and results. Finally, students will complete a written report and present a poster at a school-wide symposium.

Burnout and Curriculum Engagement in Doctor of Pharmacy Students as Related to Self-Reported Performance. Shadi Doroudgar, Touro University California, Vanishree Rajagopalan, Touro University California, Margaret Schulte, Touro University California, Bobby Wu, Jasmeet Singh, Tatiana Ouabo, Diana Nguyen, Jordon Mar, Fu Sang Luk, Manpreet Kaur, Jacob Long. This study assessed burnout and engagement in pharmacy students and investigated their relationships to perception of academic ability and performance. Burnout measures unresolvable stress, while engagement measures feelings of positivity and fulfillment. Both can influence academic performance. Several studies have measured the prevalence of burnout and engagement in health professional graduate students. This is the first study that focuses on burnout in pharmacy students and its relationship to perception of academic ability and performance. First and second year pharmacy students enrolled in didactic coursework at Touro University California-College of Pharmacy completed a Qualtrics survey with three validated scales in May 2017. The Maslach Burnout Inventory (MBI) assessed burnout using three subscales: Emotional Exhaustion (MBI-EE), Cynicism (MBI-C), and Professional Efficacy (MBI-PE). The School Attitude Assessment Survey-Revised (SAAS-R) assessed students' self-perception of academic ability and achievement. The Utrecht Work Engagement Scale (UWES) measured student engagement in three subscales: Vigor (UWES-V), Dedication (UWES-D), and Absorption (UWES-A). Data included 166 student responses (86.9\% response rate) and 96 of these participants $(59 \%)$ responded to the self-reported GPA question. UWES-V $(\mathrm{p}<0.040)$ was negatively correlated with self-reported GPA whereas UWES-D $(p<0.047)$ was positively correlated with self-reported GPA. Higher MBI-EE $(p<0.001)$ and lower MBI-PE $(p<0.001)$ were negatively associated with SAAS-R. UWES-D was also positively associated ( $p$ $<0.003)$ with higher SAAS-R. As expected, greater dedication (UWES-D) was associated with greater self-reported GPA and selfperception of academic ability (SAAS-R). Students with greater selfperception of abilities, reported higher emotional exhaustion (MBI-EE) and lower professional efficacy (MBI-PE).

Capstone Projects: Student-Driven Scholarly Activities Applying Basic Science Skills within a College of Pharmacy Curriculum. Edward Fisher, Marshall B. Ketchum University, Zhijun Wang, Marshall B. Ketchum University, Charitha Madiraju, Marshall B. Ketchum University, Herman Johannesmeyer, Marshall B. Ketchum University, Joshua A. Garcia, Marshall B. Ketchum University, Elvin Hernandez, Marshall B. Ketchum University. In light of the competitive market for graduating student pharmacists, efforts in distinguishing themselves are enhanced through documented academic excellence or through participation in co- and extra-curricular opportunities. Marshall B. Ketchum University's College of Pharmacy (MBKU-COP) developed a series of courses designed to provide opportunities for students to participate in and conduct scholarly activities in pharmaceutical sciences and other health-related science disciplines. This three-course sequence is known as Capstone Project I-III (PHM 680, PHM 750, PHM 751), which accounts for 2.0 units. Objectives include: 1 . Facilitating student-driven scholarly activities, 2 . Assisting students in designing a research project that may incorporate basic science research, educational research, community-based research, clinical research, literature review, or other forms of scholarship, 3. Facilitating the execution of a research plan including but not limited to data collection, data analysis, drawing research conclusions or presentation of the results. By accomplishing these objectives, graduates will acquire a competitive advantage in performing scholarly activities compared to many other pharmacy school graduates, relative to seeking opportunities in residency, industry, or academia. Research project selection is determined by a "ranking and match" procedure, in which faculty present various research opportunities and students prioritize their selections via Qualtrics ${ }^{\circledR}$. Student preferences are randomized and subsequently matched to faculty mentors. Students and faculty mentors then embark on completing the scholarly activities per the threecourse sequences' requirements. Forty P2 students have passed the first course, Capstone Project I, with scholarly projects currently underway in Capstone Project II, and in the future in Capstone Project III.

Clinic to Kitchen: Blending Pharmaceutical Science and Pharmacy Practice with Culinary Medicine. Malav S. Trivedi, Nova Southeastern University, Kimmi Stultz, Nova Southeastern University, Jaime W. Riskin, Nova Southeastern University, David J. Mastropietro, Nova Southeastern University. Pharmacy curricula generally lack foundational nutrition education, despite well-established benefits of nutritional interventions in the management and prevention of chronic illness. Pharmacists are readily accessible and have many opportunities to utilize professional knowledge and skills to promote health awareness. The College of Pharmacy has designed an elective course, Clinic to Kitchen, based on the "teaching kitchen" concept, which has previously demonstrated improved patient outcomes in other healthcare educational programs. This evidence-based nutrition course blends pharmaceutical sciences with practical and culinary experiential education, training students to implement sound lifestyle interventions, thus fundamentally improving patient care. Students progress through four modules within the course: Knowledge, Skills, Application and Implementation. The course begins with the unique pharmaceutical perspective of pathophysiology and determinants of health, therapeutic aspects of nutrition, biochemical theories, and nutrition assessment. Next, students develop culinary skills in a professional kitchen setting, while discussing methods to create affordable and practical meals. Students then integrate foundational knowledge and skills to assess patient cases, simulate patient interactions, and develop nutrition-focused, patient-centered care plans. Finally, students exert their innovative strength, independently designing, planning and executing a community outreach event, under the mentorship of both pharmaceutical science and pharmacy practice faculty. This course provides pharmacy students tools to confidently, effectively, and appropriately engage and counsel patients regarding the benefits of nutrition and mindful eating for long-term health and well-being. The integration of nutritional science, pharmacy practice and culinary education furthers opportunities for interprofessional education, collaboration, and teambased health care delivery, ultimately improving patient care.

Collaborating to Combat the Opioid Crisis. Robert B. Stanton, Marshall University, Brittany L. Riley, Marshall University, V. Blair Journigan, Marshall University, Charles Babcock, Marshall University, Eric R. Blough, Marshall University, Kimberly A. Broedel-Zaugg, 


\section{American Journal of Pharmaceutical Education 2018; 82 (5) Article 7160.}

Marshall University, Sarah Plummer, Marshall University, Casey Fitzpatrick, Marshall University. West Virginia has the highest drug overdose rate in the country, and drug overdose is the leading cause of mortality for West Virginians under the age of 45. Additionally, one out of every eight newborns in Cabell County, WV is born drug exposed, 40 times higher than the national average. Marshall University's School of Pharmacy (MUSOP) is proactive in combatting these issues. MUSOP collaborates with numerous local and national organizations with four key goals in mind: (i.) prevention, (ii.) harm reduction, (iii.) treatment, and (iv.) research. APhA-ASP provides interactive Generation Rx presentations to local schools and assists the Cabell County Substance Abuse Prevention Program with Drug Take Back to prevent abuse. MUSOP works with the University to provide specialized training in the Screening, Brief Intervention, and Referral to Treatment (SBIRT) model to help identify, reduce, and prevent abuse and dependence of alcohol and illicit drugs. A faculty member, along with students, assists the Cabell-Huntington Health Department in a naloxone training and distribution program in addition to needle exchange. Faculty members present and train others in the Voluntary Long Acting Reversible Contraception program used by the county's family court and the state prison system. A faculty member, along with students, educates consumers at Prestera Center, a facility that provides addiction recovery services including MedicatedAssisted Treatment (MAT) with naltrexone and buprenorphine/naloxone. Lastly, MUSOP has numerous ongoing research projects to combat this epidemic, including the development and evaluation of novel menthol receptor antagonists for neuropathic pain as well as a harm reduction survey.

Collaboration Across Departments and University Stakeholders Reduces Impact of the Decreasing PharmD Applicant Pool. McKenna Salazar, California Health Sciences University, Sree N. Pattipati, California Health Sciences University, Patty Havard, California Health Sciences University, William Ofstad, California Northstate University, Wendy Duncan, California Health Sciences University, Delwar Hussain, California Health Sciences University, David G. Fuentes, California Health Sciences University, Jeremy Hughes, California Health Sciences University. Background: California Health Sciences University (CHSU) is committed to developing compassionate, highly trained, intellectually curious, adaptive leaders capable of meeting the healthcare needs of the future through a performance-based education. We seek to matriculate qualified candidates in alignment with holistic admissions. Bringing sufficient, high caliber candidates requires an all-inclusive effort and collaboration from stakeholders across departments of Clinical \& Administrative Sciences (CAS) and Pharmaceutical \& Biomedical Sciences (PBS), and our entire CHSU community. Methods: Admissions days, high school, college and community outreach, undergraduate internships, community events, open houses, diversity grants, community partnerships and PharmD student ambassadors inspire diverse students from our region to commit to healthcare careers that serve our region. CAS and PBS teamed-up with students and volunteers from the Central Valley to implement these initiatives, supporting immediate and future admission goals. Results: Since Fall 2017, five student admissions days occurred where colleagues across CAS and PBS interviewed and interacted with PharmD candidates. Twenty-seven additional campus events featuring team-based learning (TBL), educational games, and panel speakers were held. Community health outreach involving both departments served 975 patients from the local underserved population. Open houses, college and high school visits included faculty engagement, activities with compounding, patient assessment, and medication therapy management. Community college interns in our Prospero program also served as mentors to Latino, nonnative, English-speaking students. Future Implications: These collaborative interventions help boost admissions during lows in the PharmD student applicant pool, as well as positively unify the CAS, PBS and entire CHSU community.

Collaboration Among Pharmacy Practice and Pharmaceutical Sciences Optimizes Patient Care. Michael G. Kendrach, Samford University, Peter J. Hughes, Samford University, Gregory S. Gorman, Samford University, Kimberley W. Benner, Samford University. Introduction: Within pharmacy education, a divide between Pharmacy Practice and Pharmaceutical Sciences often exists. While combining these disciplines for the students' education is imperative, this collaboration also can advance patient care. ACPE Accreditation Standard 4 advocates exposing students to knowledge, skills, abilities, behaviors, and attitudes necessary to demonstrate innovation and entrepreneurship, among others. Additionally, Standard 4 endorses collaboration whereby students interact with individuals from a broad range of disciplines. At our institution, practice and science have integrated efforts on many research projects in order to achieve this goal. Objective: Describe the collaboration of Pharmacy Practice and Pharmaceutical Sciences faculty, students, researchers and practitioners to improve patient care. Description: The Samford-McWhorter faculty from both Pharmacy Practice and Pharmaceutical Sciences departments collaborate on multiple research interests. Currently, we have affiliations with three health systems to conduct translational research with the goal of improving patient care. These projects include assessing: extemporaneously compounded oral suspension and injection stability; multi Y-site compatibility; antiviral activity of antimicrobials; as well as analysis of antibiotic removal by dialysis. Faculty, practitioners, and students have collaborated on innovative projects that have resulted in improved patient care. Many projects have been published and shared with the larger scientific community. Conclusion: Our institution has integrated research across the practice and science departments leading to results that are directly applied to patients at partner sites. Collaboration across pharmacy disciplines can provide unique opportunities for practitioners, researchers, and students, leading to improved patient care.

Collaboration at Midwestern University Chicago College of Pharmacy for the Big Three: Teaching, Service, Scholarship. Nancy Fjortoft, Midwestern University/Downers Grove, Susan R. Winkler, Midwestern University/Downers Grove, Shridhar V. Andurkar, Midwestern University/Downers Grove, Kathryn Wdowiarz, Midwestern University/Downers Grove, Medha D. Joshi, Midwestern University/ Downers Grove, Brooke Griffin, Midwestern University/Downers Grove. The College consists of two departments; pharmacy practice and pharmaceutical sciences. The departments are housed together in the same office suite, which has made collaborations both organic and purposeful. Successful collaborations in the areas of teaching, service, and scholarship are highlighted. In the area of teaching, collaborations between the two departments and the Pharmacology Department in integrating course sequences (Pharmacotherapeutics, Pharmacology, and Medicinal Chemistry) have helped streamline the course content and removed redundancies to ensure optimal student learning. Faculty members from both departments have formed interdisciplinary teams to pursue Scholarship of Teaching and Learning (eg, a web-based game). In the area of service, pharmaceutical science faculty routinely observe the delivery of clinical pharmacy services by pharmacy practice faculty. This has offered additional opportunities for communication, research collaboration, and incorporation of clinical perspectives 


\section{American Journal of Pharmaceutical Education 2018; 82 (5) Article 7160.}

in didactic teaching in pharmaceutical science courses (i.e., case studies). In the area of scholarship, several interdepartmental research teams have been formed in the last decade, bringing together the strengths of clinical and basic science faculty. This has led to successful research endeavors, posters, publications, and the purchase of university-funded laboratory equipment. Intramural and extramural grant funding continues to support ongoing collaborations. With these collaborations, teams have been able to study and contribute to innovative translational research in the areas of infectious diseases and women's health. Successes and challenges of each will be reviewed and faculty perceptions will be highlighted. Future plans include expanding collaborations in teaching, service, and scholarship to include other colleges within Midwestern University.

Collaborative Approaches to Enhancing Student Education. Jaime R. Hornecker, University of Wyoming, Becky Linn, University of Wyoming, Sommer D. Zarbock, University of Wyoming, Alvin B. Oung, University of Wyoming, Baskaran Thyagarajan, University of Wyoming, Michelle L. Hilaire, University of Wyoming, Reshmi L. Singh, University of Wyoming, Thanh-Nga Nguyen, University of Wyoming, Mary K. Onysko, University of Wyoming, Allison Mann, University of Wyoming, Leena D. Myran, University of Wyoming. The University of Wyoming School of Pharmacy (UWSOP), located in Laramie, Wyoming, consists of faculty members from diverse backgrounds including biomedical, pharmaceutical, social-administrative, behavioral, and clinical sciences. Collaboration among this tight-knit group enriches the work that our faculty does and enhances and broadens the education and experiences of our pharmacy students. The faculty are involved in all aspects of UWSOP administration and student educational experiences. Students experience this collaboration initially with pharmacy student candidate interviews when they have opportunities to meet with pairs of core faculty and ask questions regarding the program. Once enrolled, students are assigned to a team of faculty advisors from different disciplines that guide, encourage, and support them through their pharmacy career. The faculty advisors' role to support students ranges from curricular and career advising to co-curricular activities. Additionally, UWSOP students and faculty from multiple disciplines undergo formalized leadership training utilizing the "5 Voices" curriculum. Faculty from all disciplines, PharmD students and $\mathrm{PhD}$ candidates often work simultaneously on research, grant writing, and other scholarly endeavors. The most recent collaborative undertaking for the UWSOP is the development of a longitudinal skills course and curriculum alignment. This new curricular design aims to reinforcing knowledge application through integrating all course content into the patient care process. This endeavor has required focused collaboration and discussion of the curriculum amongst the entire faculty, with the end goal being a revamped curriculum for future UWSOP classes that will produce quality graduates ready to participate in direct patient care.

Collaborative Efforts between Departments in Teaching, Research, and Service in a Block-System Three-Year Pharmacy Program. Gary M. Levin, Larkin University, Sandra Benavides, Larkin University, Subrata Deb, Larkin University, Joshua Caballero, Larkin University. Larkin University places a strong emphasis on collaboration between the Departments of Clinical and Administrative Sciences (DCAS) and Department of Pharmaceutical Sciences (DPS) in its block-system, threeyear Pharm.D. curriculum. It has been the goal of the founding Dean to ensure both departments collaborate in all aspects of our College including teaching (curricular and co-curricular), research, and service. Descriptive statistics were used to describe collaborative efforts between departments during the first two years of the program. There is integration of faculty from both departments in 17 didactic disease-based course blocks and three non-disease-based blocks. Faculty from DCAS and DPS meet before starting each block to discuss the delivery and active learning strategies of educational content. Examples of topics discussed include current medications used, pharmacokinetic parameters, avoiding duplication of material, and ensuring an increase in the expectation of core concepts as blocks progress. Among disease-based blocks, approximately $23 \%$ of the material is taught by the DPS faculty. In two years, faculty from both departments have collaborated in educational research presented at national meetings. Topics include optimal/reasonable/quantifiable faculty evaluations, impact of active learning in pharmaceutical calculations, pharmacist patient care process evaluation tool, and gamification of lectures to medical residents. Additionally, the first interdepartment manuscript was recently published in a Medline Indexed journal. Grading of student assignments during introductory pharmacy practice experiences are also shared between departments. International collaborations (eg, student exchange program), and interdepartment advanced pharmacy practice experiences have been developed. The Executive Council supports collaborative endeavors between the DCAS and DPS.

Complementary Approaches with a Common Mission: Integrating the Pharmaceutical Sciences with Pharmacy Practice. Noha Salama, St. Louis College of Pharmacy, Jasmina Profirovic, St. Louis College of Pharmacy, Golden L. Peters, St. Louis College of Pharmacy, Lara C. Kerwin, St. Louis College of Pharmacy, Peter D. Hurd, St. Louis College of Pharmacy, Brenda L. Gleason, St. Louis College of Pharmacy, Bruce Canaday, St. Louis College of Pharmacy, Rhonda Bilger, St. Louis College of Pharmacy, Tricia M. Berry, St. Louis College of Pharmacy. Based on institutional strategic plans, St. Louis College of Pharmacy employed an evidence-based, backward design approach to a comprehensive curricular revision. To prepare highly competent graduates capable of providing optimal patient care in the 21 st century, integration of disciplines and application of knowledge and skills to therapeutic decision-making were some of the faculty's guiding principles to curricular reform. Design and implementation of our new curriculum brought together faculty with complementary areas of expertise from our two academic departments - Pharmaceutical \& Administrative Sciences and Pharmacy Practice. Our Integrated Pharmacotherapy (IP) series, comprised of 12 course modules over 5 semesters, integrates pathophysiology, pharmacology, medicinal chemistry, pharmacotherapy, self-care and pharmacogenomics. The Pharmacy Practice Skills Lab (SL) series, with 5 semester-long courses, aligns with and allows for practice and application of topics within the IP series. Concepts from other courses (e.g., pharmacy calculations, pharmaceutics, information mastery, health system management) are also reinforced in the IP and SL series and provide opportunities to deepen student comprehension and application. For example, students in SL can polish their compounding skills while enhancing and applying related scientific knowledge as they are presented with dosage forms and patient cases tailored to the disease state covered in the IP series. The curricular shell, examples of course syllabi, practice opportunities, and assessment tools will be highlighted. Faculty experiences with the collaborative curricular design and implementation process will be described, including successes and challenges of a highly-integrated curricular model and proposed curricular revisions to address some of the identified challenges.

Complementary Tracks Connecting the Pharmaceutical Sciences and Pharmacy Practice: Encouraging Research for PharmD Students. Janet K. Astle, Duquesne University, David A. Johnson, Duquesne University, Peter L.D. Wildfong, Duquesne University. Background: Connecting the basic sciences and pharmacy practice is encouraged 


\section{American Journal of Pharmaceutical Education 2018; 82 (5) Article 7160.}

by the 2016 ACPE Guidelines, which recommend "broadening student experiences" (Std. 9e), and linking "scientific understandings and patient care" (Std. 10d). Direct efforts to blend science and practice can be helped by establishing a formal concentration, the implementation of which can benefit from an extant complementary graduate program. Broader experiences during pharmacy education will enrich the profession by providing future pharmacists with a more complete perspective. Description: In addition to its PharmD program, the School offers advanced degrees in pharmaceutics, pharmacology, medicinal chemistry, and the social/administrative sciences through the Graduate School of Pharmaceutical Sciences. Basic sciences faculty introduce their current research throughout the PharmD curriculum, often piquing the interest of professional students. Those seeking volunteer laboratory opportunities are encouraged to complete the Research Concentration, which couples tailored elective coursework with formal faculty advisement on laboratory projects. PharmD students enrolled in the Research Concentration may elect to complete a commensurate Bachelor of Science in Pharmaceutical Sciences (BSPS). Financial support for PharmD students to present research at national science conferences is provided as a conduit to co-authorship of peer-reviewed research articles and abstracts. Implications: When current scientific research is interspersed throughout PharmD curricula, students are inspired to better understand how that research underpins clinical aspects of their profession. The Research Concentration is enabled by pairing with research laboratories administered through the Graduate School, providing a pathway that allows PharmD students to earn a commensurate BSPS degree, advised by researchers in the field.

Connecting Pharmaceutical Sciences and Pharmacy Practice: Collaborations at Purdue University. Jane E. Krause, Purdue University, Richard M. van Rijn, Purdue University, Tony R. Hazbun, Purdue University. Objectives: 1) To describe a three-hour P1 onecredit, service-learning elective course (Drug Abuse/Addiction Education) and a P1 laboratory (Antibody-Based Diagnostic Tests). Both are co-taught by professors from two disciplines (Pharmacy Practice and Medicinal Chemistry \& Molecular Pharmacology) using an integrated instructional format. The Drug Abuse/Addiction Education service-learning course connects pharmacology with practice. The laboratory illustrates two types of diagnostic tests by integrating principles of immunology and practice/patient care. 2) To assess the impact of the service-learning course on perceptions of the students. 3) To assess the impact of the laboratory on knowledge of the students. 4) To evaluate feedback on both teaching collaborations. Methods: Students in the service-learning course reflected on the experience by completing a 16 item anonymous questionnaire utilizing a five-point Likert scale at the start and end of the course. Students in the laboratory completed a 10 item multiple-choice quiz prior to and immediately following the laboratory. Feedback on instruction was obtained. Results: 32 students were enrolled in the service-learning course and results indicate a statistically significant change in students' perceptions (14 items, $\mathrm{p} \leq 0.05$ ). 148 students participated in the laboratory and results indicate a statistically significant $(\mathrm{p} \leq 0.05$ ) improvement in 9 (of the 10 quiz items) and in total quiz scores. Mean quiz score increased by $32.9 \%$ following the laboratory. The interdisciplinary/team teaching approach was well received by the students in both experiences. Implications: Pharmaceutical sciences serves as a means for helping students understand pharmacy practice/patient care concepts.

Connecting Pharmaceutical Sciences and Pharmacy Practice through the Development of a Student Organized PharmDPharmTox Research Symposium. Paria Sanaty Zadeh, Melgardt M. de Villiers, University of Wisconsin-Madison, Paul R. Hutson,
University of Wisconsin-Madison, Kenneth S. Niemeyer, University of Wisconsin-Madison, Barry E. Gidal, University of Wisconsin-Madison, Lindsey S. Skubitz, Katie S. Windschitl. Objective: To support research and the scholarly contributions of developing pharmacy professionals, and to provide an opportunity for students and researchers to network and explore future research opportunities our students developed an annual PharmD and Pharm-Tox Research Symposium and poster competition. Methods: UW-Madison is somewhat unique because in addition to PharmD students and residents we also offer an undergraduate BS degree in Pharmacology-Toxicology (PharmTox). Students from these groups participating in research with a faculty mentor are encouraged to submit an abstract, which is peer-reviewed by the student symposium leaders to ensure consistency in format. On the day of the symposium posters are judged by a team of SOP clinical and pharmaceutical science faculty, using a student developed tool that evaluates both organization, content, visual clarity and their ability to transmit this information. The top 3 presenters are awarded a modest travel award to facilitate attendance at a national scientific or professional meeting of their choice. Outcomes: Over the past three years the symposium have showcased research completed by PharmD students, Pharm-Tox undergraduates, and pharmacy residents in the Madison area. Students gained scientific writing experience and enhanced presentation skills. By fostering these essential skills, students felt better prepared for national poster presentations and post-graduation opportunities. The forum also provided an opportunity for networking and career path discussions. Overall, the symposium has served as a dedicated event that promotes scholarly activity early in students' pharmacy careers, encourages idea exchange on pharmacy research, and promotes a culture of future scholarly approach to practice.

Connecting PharmD Students to Research in Pharmaceutical Sciences through a Research Capstone. John R. Reynolds, Northeastern University, Gregory M. Miller, Northeastern University, Diomedes E. Logothetis, Northeastern University, Margarita V. DiVall, Northeastern University, Jennifer Kirwin, Northeastern University. Background: Recent calls for personalized education opportunities led our school of pharmacy (SOP) to create a new research capstone course for PharmD students. This expanded flexibility in the way students could meet the University's capstone requirement. Prior to this change, all students completed an economic evaluation as their capstone topic with a Pharmacy Practice faculty member. Methods: The 4-credit research capstone course allows students to work alone or in groups with one or more faculty advisor(s) on a project of mutual interest. Information on types of projects and research discipline was collected from internal SOP records of proposed topics for classes of 2018 and 2019. Results: For the class of 2019, 140 students submitted capstone proposals for 62 discrete projects. Twenty-one (34\%) of these identified a faculty mentor from the Pharmaceutical Sciences (PS) department. This was an increase from six PS projects for the class of 2018. Combined data for both cohorts showed involvement from six PS faculty ( $43 \%$ of the department) who worked with 55 students $(20 \%)$. Nine of the PS projects (33\%) were identified as bench/lab research, while the rest were applied in nature. Conclusions: Introduction of a more flexible capstone model allowed many PharmD students to work with faculty on projects related to PS. Distribution of projects may have been limited by faculty resources for student research or student interest in PS topics. Efforts to increase collaboration among faculty from different departments are underway.

Connecting the Sciences and Pharmacy Practice through Student Engagement. Terri L. Warholak, The University of Arizona, Elizabeth A. Hall-Lipsy, The University of Arizona, Marion K. Slack, The University of Arizona. Background: The ideal situation for every college 


\section{American Journal of Pharmaceutical Education 2018; 82 (5) Article 7160.}

or school of pharmacy is to be well connected with its community. Such a connection can bridge the gap between academia and practice to assure that learning is grounded in reality and to assure that research and other programs respond to the changing nature of the profession. Additionally, through their collaborative work with students, practitioners and community members have access to university services including library resources and research expertise. Purpose: The purpose of this presentation is to demonstrate how programs based on student engagement can connect interprofessional and research sciences to practice. Methods Examples of student programs that connect the College to pharmacy practice will be described. Results: Three programs that connect science to practice were identified: Quality Improvement Projects, Student Research Projects, and Interprofessional Regulatory Science Programs. Quality Improvement Projects are required for all students; working in groups of 4, the students connect with a practitioner to address an improvement issue at their practice site. Student Research Projects, which are likewise required, connect a project advisor and a student group to address a practice problem. The interprofessional program in regulatory science is an optional program linking pharmacy, public health and law faculty and students to industry and regulators for projects and colloquia exploring the relationship between science and regulation. Conclusions: Student engagement programs can connect the College and practitioners so that the College and students benefit from practitioner experience and practitioners benefit from increased access to the university's expertise and resources.

Cultivating Interdepartmental Collaboration with the Ancient Practice of Pharmacognosy. Joseph R. Ofosu, University of Saint Joseph, Dayne A. Laskey, University of Saint Joseph, Stephen R. Slauson, University of Saint Joseph. Objective: Clinical problems stemming from daily practice often have laboratory-based solutions. We present a patient case that led to collaboration between faculty members of the Department of Pharmacy Practice and Department of Pharmaceutical Sciences and resulted in a pharmacy student research project. Scenario: Brodifacoum is a long-acting anticoagulant rodenticide which can cause prolonged morbidity and possible mortality following large ingestions. Treatment includes long-term daily doses of vitamin K1 (VK) to prevent rebound coagulopathies. A practice faculty encountered a patient in the hospital whose course was complicated by inability to afford treatment. A science faculty agreed to help determine whether kale could be practically used to extract enough VK to treat a patient. Working with a pharmacy student on a 6 week APPE research rotation, a laboratory protocol was developed to test a potentially patient-friendly kale extraction method. High-Performance Liquid Chromatography was used to evaluate the VK content of the kale extract. Preliminary results suggested that $15.5 \mathrm{mcg}$ of VK can be extracted from every 1 gram of kale by concentrating into a lipophilic layer. Therefore, a therapeutic dose for an adult patient would require extraction of over $3 \mathrm{~kg}$ of kale per day. Conclusions: In this complementary approach, a patient treatment question connected faculty from Pharmacy Practice and Pharmaceutical Sciences to accomplish a common goal, resulting in a meaningful APPE student project and multiple scholarship opportunities.

Data-Driven Research Initiatives at Idaho State University College of Pharmacy: Synergizing Pharmaceutical Sciences and Pharmacy Practice. Elaine Nguyen, Idaho State University, Karl J. Madaras-Kelly, Idaho State University, Vaughn L. Culbertson, Idaho State University, Dong Xu, Idaho State University. With the ever-increasing amount of available scientific and healthcare data, state-of-the-art clinical and translational research has entered the big data era. Idaho State University
(ISU) College of Pharmacy (COP) recognizes this trend and is embracing big data-driven research areas that synergize pharmaceutical sciences and pharmacy practice. Here we showcase a few examples of emerging research projects being carried out at the ISU COP: (1) TargetSearch, an integrative cross-database bioinformatics web service for drug discovery, drug re-purposing, and drug safety research; (2) GPI-FAERS, GPIencoded FDA adverse events reporting system for improved data quality and query performance; (3) MSBIS, a multi-step biomedical informatics screening approach for identifying medications that mitigate the risks of metoclopramide-induced tardive dyskinesia; (4) ATS, an integrated computational approach for assessing and predicting drug-induced anticholinergic risks; (5) Serotonin Expanded Bioactivity Matrix (SEBM), off-target serotonergic drug effects evaluated by a bioinformatics molecular analysis of serotonin syndrome reports; (6) comparative effectiveness of influenza vaccines; (7) impact of remote monitoring in chronic care management, (8) Implementation of standardized provider audit and feedback reports designed to improve diagnostic and treatment performance. The ISU COP is also establishing a bioinformatics and health outcomes research core facility to provide big data computing infrastructure and HIPAA-compliant data security. We anticipate that these new research initiatives and resources will facilitate collaborations among our pharmaceutical sciences and pharmacy practice faculty and further enhance interdisciplinary and translational research at ISU.

Data Mining Applications to Predict Students' Performance on the North American Pharmacist Licensure Examination (NAPLEX). Jonathan Hernandez-Agosto, University of Puerto Rico. Introduction: Knowledge discovery in databases (KDD) methodology has been used recently to automatically generate predictions on student performance. This approach, also known as data mining, was used in this study to determine: 1) the most relevant predictors of students passing NAPLEX on first attempt, and 2) which KDD technique provides a better classification result in predicting students passing NAPLEX on first attempt. Methodology: This cross-sectional study design with analytical scope, considered as main data sources, admission variables highlighted as predictors of student success, the National Association of Boards of Pharmacy (NABP) scores reports on NAPLEX, and Pharmacy Curriculum Outcomes Assessment (PCOA) results. Pearson correlation analysis and four basic stages of the KDD method were performed to a data file of $37(n=37)$ valid student records. Four classification algorithms (Decision Tree, Logistic Regression, Multilayer Perception, and Naïve Bayes) were evaluated as predictive models. Results: Correlation results showed a moderate positive relationship between PCOA scores and NAPLEX performance $(r=.632$, $\mathrm{p}<.000)$. Naïve Bayes (accuracy $=94.6 \%$ ) and Decision Tree C4.5 (J48) (accuracy $=91.9 \%$ ) classification algorithms outperformed other classifiers when predicting passing the NAPLEX on first attempt. Decision Tree provided a PCOA Area 1 cutoff of 308 of being predictive of student passing. For scores below this threshold, PCAT score provided additional predictive power in predicting NAPLEX performance. Implications: KDD techniques hold the potential to help pharmacy educators and administrators to identify relevant factors that contribute to student passing NAPLEX on first attempt, to improve curricular decisions, and student academic performance.

Degree Programs Offered by The University of Georgia College of Pharmacy. Yujun Zheng, The University of Georgia, Somanath Shenoy, The University of Georgia, Henry Nolan Young, The University of Georgia, Trina J. von Waldner, The University of Georgia, Michael G. Bartlett, The University of Georgia, Catherine A. White, The 


\section{American Journal of Pharmaceutical Education 2018; 82 (5) Article 7160.}

University of Georgia. The University of Georgia College of Pharmacy has a Bachelor of Science in Pharmaceutical Sciences program as well as a dual Bachelor of Science in Pharmaceutical Sciences and Master of Science in Pharmacy program. The Bachelor of Science program in Pharmaceutical Sciences was established in 2013 and has graduated 34 students. The dual BS/MS program was approved in 2016 and admitted 8 students in 2017. Currently these is one PharmD dual degree program, PharmD/Master of Public Health which was implemented in 2010 and has enrolled 20 students. The College also offers residencies in Critical Care, Ambulatory Care, Oncology, Pediatrics, Emergency Medicine, Clinical Pharmacy and Community Pharmacy. The Master of Science and Doctor of Philosophy programs include Pharmaceutical Sciences, International Biomedical Regulatory Sciences (MS only), Clinical and Experimental Therapeutics, Toxicology and Pharmaceutical Health Science Policy and Outcomes. Many PhD students in the Pharmaceutical Sciences Program opt to complete the MS in International Biomedical Regulatory Sciences program. Faculty in the Department of Pharmaceutical Sciences recently received a grant to develop 3 online graduate courses regarding the drug discovery/ development process. These courses will be available to students in the BS/MS program and in all Master of Science and Doctor of Philosophy programs. These will be the first courses developed that will cross all disciplines within the College.

Design \& Integration of Pharmacy Curricula across Disciplines: The University of Utah College of Pharmacy (UUCOP) Experience. Donald K. Blumenthal, The University of Utah, Jaclyn Winter, The University of Utah, Alisyn May, The University of Utah, Hanna Raber, The University of Utah, Craig P. Henchey, The University of Utah, Heather A. Nyman, The University of Utah, Patricia L. Orlando, The University of Utah. Curricula are evolving within US pharmacy schools in response to ACPE standards 1-4 that propose students should no longer be expected to make their own connections between scientific concepts and clinical applications. Instead, curricular designs favor integrated approaches to connect basic science with pharmacy practice in real-world patient care applications. The new UUCOP integrated curriculum is designed to make these connections and provide an interdisciplinary professional training program for future clinical scientists, educators and practitioners. As part of major curriculum revisions in 2015, the UUCOP developed a series of three Integrated Pharmacotherapeutics courses combining pathophysiology, medicinal chemistry, pharmacology/toxicology, pharmacokinetics, and therapeutics during the P2 and P3 years. Two course masters, from pharmacology and pharmacotherapy, oversaw implementation of each course. Intracourse organ-based modules paired basic science faculty with clinical faculty as leaders and content overseers. Pharmacotherapeutics courses are supported by weekly two-hour recitations, designed to utilize active, team-based instruction to challenge students to apply knowledge and skills. Recitation leaders consist of basic science and clinical faculty pairs. Implementation thus requires that faculty from all UUCOP departments work together to oversee and deliver this curriculum. The College uses surveys of students, course masters, preceptors, and employers (based on CAPE Outcome scales) to monitor curriculum effectiveness. Results will be compared between old baseline data and using P2/P3 PCOA scores. The faculty will quantitatively and qualitatively assess the overall new curriculum effectiveness with the 2019 graduation class.

Design of an Integrated Pharmacy Laboratory Sequence. Omathanu P. Perumal, South Dakota State University, James R. Clem, South Dakota State University, Jane R. Mort, South Dakota State University, Teresa M. Seefeldt, South Dakota State University. Content integration and active learning are two elements in Standard 10 of the Accreditation Council for Pharmacy Education Standards 2016. To address these elements, curricular change was undertaken to enhance integration of content between pharmaceutical sciences and pharmacy practice content and increase emphasis on active learning. Curricular integration efforts started with a project focused on incorporation of interdepartmental teaching activities into didactic courses taught with active learning pedagogies. This evolved into a laboratory course sequence expanding integration of disciplinary content and application to practice. The integrated pharmacy laboratory sequence includes one course in each year of the pre-APPE curriculum. Laboratory activities will involve integration of content from all courses taught during that year as well as reinforcement of previously learned content. These laboratory courses will focus on development of critical thinking and problem-solving skills by utilizing a variety of active teaching and learning methods including case-based learning, simulations, and wet lab exercises. The activities are being developed by teams of faculty with expertise in pharmaceutical sciences and pharmacy practice, and the courses will have a coordinator from each department in order to model integration. Two disciplinary teaching laboratory spaces are being remodeled in order to facilitate integration and create dynamic learning environments focused on small group interaction. One lab will be a pharmacy care laboratory, and the other will be a compounding laboratory. Through these improvements in instruction, students will be effectively engaged in active, collaborative learning that integrates information into a practice-based experience.

Developing and Delivering an Integrated Curriculum through Collaboration of Pharmaceutical Science and Pharmacy Practice Faculty. Marie A. Chisholm-Burns, The University of Tennessee, Vivian S. Loveless, The University of Tennessee, Leslie A. Hamilton, The University of Tennessee, Stephanie J. Phelps, The University of Tennessee, Dawn E. Havrda, The University of Tennessee. The University of Tennessee Health Science Center College of Pharmacy worked over 2.5 years to develop the 4-UT curriculum, which integrates and aligns pharmaceutical sciences (medicinal chemistry, pharmacology, pharmaceutics) with pharmacy practice (clinical and translational sciences, health outcomes). The inclusive and collaborative planning process involving all disciplines and resulted in a curriculum that uses an integrated organ-system approach combining physiology, pathophysiology, medicinal chemistry, pharmacology, and therapeutics. A longitudinal skill-based course combines clinical simulation and interprofessional education to allow students to develop patient care skills in team environments. First-year students learn about application of pharmaceutical science to pharmacy practice in a state-of-the art simulation center. The first-year begins with layered-learning introductory pharmacy practice experiences where students interact with patients and healthcare teams. After 2.5 years of coursework, students hone their skills through 1.5 years in advanced pharmacy practice experiences to be practice and team-ready pharmacists. The 4-UT curriculum began in Fall 2017 with 181 first-year student pharmacists. In formative assessments, students remarked positively that integration within and between courses helped them understand basic science concepts and applicability to pharmacy. Less students in the new curriculum earned a D or F in fall coursework with early introduction of integration compared to the old curriculum. Student pharmacists exhibited critical thinking skills in solving patient problems in the first year and felt confident in their knowledge base. Assessment of student performance and continuous quality improvement to enhance the students' educational experience will be discussed.

Development of a Collaborative and Interdisciplinary Lyme Disease Research Center in Rural New York. Eric Hoffman, Binghamton University, The State University of New York, Ralph M. Garruto, 


\section{American Journal of Pharmaceutical Education 2018; 82 (5) Article 7160.}

Binghamton University, The State University of New York, Gail B. Rattinger, Binghamton University, The State University of New York, Gloria E. Meredith, Binghamton University, The State University of New York, Sarah Lynch, Binghamton University, The State University of New York, Wesley D. Kufel, Binghamton University, The State University of New York. Background: Lyme disease is an endemic infectious disease and a major public health threat in rural Upstate New York. Binghamton University School of Pharmacy and Pharmaceutical Sciences (BUSOPPS) is well-positioned and has the resources to engage in high-quality, collaborative research endeavors with other departments at Binghamton University (BU) to target Lyme disease as an emerging research area to serve the rural Upstate New York area. Objective: To develop an interdisciplinary, translational Lyme disease research center at BU focused on these primary areas: diagnostics and treatment; ecology, epidemiology and risk modeling; public health education and outreach. Methods: BUSOPPS has collaborated with the Department of Anthropology at BU and our interprofessional research physician partners at Upstate Medical University located in Syracuse, NY to develop a Lyme disease research center. The proposed research center has developed over twenty research proposals involving diagnostics, management, epidemiology, and public health outreach. BUSOPPS faculty within the departments of pharmacy practice and pharmaceutical sciences provide the necessary strengths and collaborations to carry out these proposals through the combination of direct clinical experience and extensive laboratory research. A common biobank of acute and chronic Lyme positive samples is in development for researchers to conduct proposed projects. Multiple extramural funding opportunities are being sought to support the long-term goals of the Lyme disease research center. Anticipated Early Results: Establishment of this interdisciplinary, translational Lyme disease research center at BU has led to extensive interdepartmental as well as cross university collaborations that will lead to robust scholarship and successful scientific discovery.

Development of an Interdepartmental Objective Structured Clinical Exam (OSCE) as Part of a Pharmacotherapeutics Course. Ka L. Hong, Wilkes University, Ajay K. Bommareddy, Wilkes University, Vicky Shah, Wilkes University, Thomas S. Franko, Wilkes University. Objective: To describe the development and implementation of an OSCE incorporating both pharmacy practice and pharmaceutical science into a Pharmacotherapeutics course. Description: The Nesbitt School of Pharmacy at Wilkes University is located in Northeastern Pennsylvania and follows a $2+4$ model. The class size in the professional program is seventy two students. Students traditionally complete an OSCE as a capstone assessment at the end of each semester through their Pharmacy Care Lab series. Students in PHA 521, Pulmonary Pharmacotherapeutics, indicated a desire for additional active learning exercises in the course. Instructors from the pharmacy practice and pharmaceutical sciences departments collaborated to develop an OSCE which combined the therapeutics, pharmacology and medicinal chemistry of asthma. Students identified a medication-related problem from a patient chart and communicated their concern to a physician. The physician inquired about the scientific basis for the recommendation. As this was a pilot program, the result of the OSCE counted as $2.5 \%$ of each student's final grade. Each student was evaluated by a faculty member of the School of Pharmacy not associated with the course. Analysis of the results demonstrated that students were able to successfully integrate the science and treatment of asthma and communicate their rationale to a physician. The data collected, combined with student and peer feedback of the event, will enable/promote further enhancement of this assessment method for future classes.
Drug, Action, Structure, and Therapeutics (DAST): A Collaborative Didactic Course Series. Carmita A. Coleman, Chicago State University, Mohammad Newaz, Chicago State University, Betty Vu, Chicago State University, Mohammad Tauseef, Chicago State University, Mohd Shahid, Chicago State University, Lalita Prasad-Reddy, Chicago State University, Ayesha Khan, Chicago State University, Alexander Kantorovich, Chicago State University, Elmer J. Gentry, Chicago State University, Hafeez Faridi, Chicago State University, Luba Burman, Chicago State University, Yolanda M. Hardy, Chicago State University. Background: Since its inception, the College has made intentional efforts to integrate Pharmaceutical Sciences and Pharmacy Practice throughout the curriculum. A key example of this integration is the Drug Action, Structure and Therapeutics (DAST) course sequence. Course description: DAST is a core course sequence that spans the second and third professional years of the program. The course, team-taught by faculty from the Departments of Pharmaceutical Sciences and Pharmacy Practice, includes pathophysiology, medicinal chemistry, pharmacology, and pharmacotherapeutics. The courses are taught in eight modules organized by organ system/disease states and consists of didactic lectures, laboratory training, workshops, and case presentations. The variety of integrated learning approaches provide students with ample opportunity to gain practical experience in pharmacy care. Course content is based on current therapeutic guidelines. Faculty within the course ensure delivery of the most effective and advanced clinical information to the students by maintaining regular communication with each other, and by staying abreast of changes in clinical practice. Objectives: Enable students to apply knowledge from each discipline in order to develop a well-rounded and practical patient care plan following the Pharmacist Patient Care Process. Implications: An integrated course enables students to connect how various disciplines impact delivery of patient care. The overarching philosophy behind DAST is the integration of pharmaceutical and clinical sciences throughout the curriculum, enabling graduates to apply and develop treatment plans that are both evidence and team-based.

Dual Degree Programs to Connect Clinical and Research Skills Training. Curtis W. Noonan, University of Montana School of Public and Community Health Sciences, Erica L. Woodahl, University of Montana, Howard D. Beall, University of Montana, Jean T. Carter, University of Montana, Donna G. Beall, University of Montana. Students admitted to the Doctor of Pharmacy program at the University of Montana may now pursue a second, research or business oriented degree during the four-year professional program. The dual degree offerings with a research focus include a Master of Science degree in Pharmaceutical Sciences and Drug Design (MS PSDD) and a Master of Public Health (MPH). In addition to their professional degree advisor, students are assigned to a dual degree advisor who helps them navigate the two curricula and degree requirements. The dual degree programs provide two tracks - one for those with a prior baccalaureate and one for those without a degree. In addition to the MPH degree, student have options to obtain a certificate in public health which includes some research training without the time and cost of pursuing a second, separate degree. To ensure student success, School faculty and administrators have worked with offices in financial aid, graduate school, and registrar to remove barriers. At this time, there are $3 \mathrm{MPH}$ students and 1 MS Pharmaceutical Sciences students enrolled in dual degree programs with at least four first year students applying to the MPH program. There are also 17 currently enrolled students pursuing the MBA/PharmD degrees. Since the inception of dual degree programs, 13 students have graduated with PharmD/MBA, and one each 


\section{American Journal of Pharmaceutical Education 2018; 82 (5) Article 7160.}

has earned a PharmD/MPH, PharmD/MS, and PharmD/PhD. These successes have been a direct reflection of the faculty collaborations between the units of the College.

Epinephrine Drug Degradation in Auto-Injector Products. Abebe E. Mengesha, Drake University, Lynn Kassel, Drake University. Epinephrine is an adrenergic medication that works on alpha and beta receptors and is used in the treatment of shock, specifically anaphylaxis. Epinephrine auto-injectors are becoming more common within the community, due to allergies as well as vaccination programs at retail pharmacies. Epinephrine auto-injectors are dated with a relatively short expiration date, sometimes as little as 18 months from production. The cost of epinephrine auto-injectors has recently increased, due to drug shortage, drug company pricing, or a combination. This project is a multi-pronged approach to the life and cost of an epinephrine auto-injector. The initial steps of the project includes development of methods for assessing epinephrine concentrations via high-performance liquid chromatography [HPLC]. Next, this project will assess epinephrine degradation in extreme element exposure, including exposure to UV light and extreme temperatures outside of the recommendations for storage of epinephrine. The third step of this project is to utilize already expired epinephrine auto-injectors, which are available in packs of two, to determine the rate of drug degradation. As the twin-pack of epinephrine auto-injectors will allow for two data points, the rate of drug degradation can be calculated past the expiration date. Information gathered from exposure to the elements will be useful in determining how long epinephrine concentrations will remain above a certain level, such as $90 \%$. Ultimately, the completion of this project may impact the shelf-life of an epinephrine autoinjector, extending the expiration dating and reducing the need for patients and family members to frequently purchase the expensive products.

Evolution of an Integrated Pharmacotherapy Sequence at The University of Texas College of Pharmacy. Bryson M. Duhon, The University of Texas at Austin, Renee Acosta, The University of Texas at Austin, Patrick J. Davis, The University of Texas at Austin. The University of Texas at Austin College of Pharmacy first instituted an integrated pharmacotherapy course sequence with implementation of the entry level Pharm.D. in 1996. Four large, multi-module courses addressed the appropriate pathophysiology, medicinal chemistry, pharmacology/toxicology and therapeutics for specific disease state. Case-based laboratories reinforced didactic content. The first major revision of the sequence in 2010 moved from students simultaneously handling multiple disease state modules to an "immersion" model with focus on a single module until completion. In 2016, the creation of a new Physiology/Pathophysiology course sequence in the first professional year allowed for removal of the pathophysiology content from the therapeutic modules. A task force undertook a review of the entire pharmacotherapy sequence for streamlining of module content as well as a redesign for Fall-2019 where each module will represent one of thirteen standalone courses. The new sequence will allow for better horizontal integration with the Nonprescription Pharmacotherapeutics sequence and other courses taught in the P2/P3 years, and will integrate the contents from our capstone Special Populations Pharmacotherapy course (i.e., pediatrics, geriatrics, pharmacogenomics) into the appropriate module. The program assessment we conduct each semester will allow for continuous monitoring of the new sequence. A number of College policies have been reconsidered; for example, student failure in a single module will not preclude progression to the next module (reducing delays) and will allow for more expeditious, targeted remediation. The new sequence will provide a better structure for faculty accountability in modular integration and course coordination.

Evolution of Collaboration within an Integrated Pharmacy Curriculum. Paul W. Sylvester, The University of Louisiana at Monroe, Michael B. Cockerham, The University of Louisiana at Monroe, Gregory W. Smith, The University of Louisiana at Monroe. The Curriculum Committee Toolkit for Addressing the 2013 CAPE Outcomes states, "The goal of curricular integration is to ensure that the intended curriculum, enacted curriculum, learned curriculum, and assessed curriculum are all aligned." Three years ago, our faculty began a collaborative effort of identifying ways to improve the clinical practice-readiness of our candidates. The Curriculum Committee evaluated the professional pharmacy program using several assessment criteria, including data from course reviews, standardized student exam scores, and student focus groups. This rigorous evaluation has resulted in a revised curriculum that reflects ongoing improvements to content integration, delivery, and assessment within the program. Last year, the clinical and basic sciences faculty collaboratively restructured the foundational courses to improve content cohesion and transition to better support new Therapeutics modules. Assessment measures have been implemented prior to the first offerings of the new Therapeutics modules to ensure that the faculty instructors within each course are routinely collaborating to enhance discipline-specific content integration and to establish and improve clinical correlations. One such measure is the implementation of a revised Continuous Quality Improvement (CQI) tool that involves an annual review of each course by all participating faculty. This CQI review encourages scheduled interaction, and utilizes the aforementioned CAPE Outcomes Toolkit with the intent of continuously improving content integration, delivery, and assessment. In summary, to ensure continuous improvement of our curriculum, assessment tools have been placed to provide real-time evaluation of the curriculum to identify and expeditiously implement needed course adjustments to optimize educational outcomes.

Fostering a Culture of Collaboration using the Four Frames Model. Cindy D. Stowe, Sullivan University, Sara Wade, Sullivan University, Walter D. Soja, Sullivan University, Dale E. English, Sullivan University, Arthur G. Cox, Sullivan University, James D. Nash, Sullivan University, Misty M. Stutz, Sullivan University, Kimberly K. Daugherty, Sullivan University, Daniel R. Malcom, Sullivan University. Sullivan University College of Pharmacy is a three-year accelerated PharmD program with two departments. Our purpose is to describe the adaptive challenge of creating a culture of collaboration supported by Bolman and Deal's Four-Frames Model. The Four-Frames Model provides a construct to understand the complexity of organizational culture. These four constructs are structural, human resource, political, and symbolic. Structurally, there was an intentional creation of a collaborative culture beginning early in the life-cycle of the College. From the human resource frame, there is collaboration between department chairs, transparency of faculty workload calculations, and a co-teaching and co-coordination of courses between departments. A political change occurred resulting in changing the course nomenclature to a unified "PHR\# course title." Faculty office location assignment is intentional having faculty members from both departments interspersed between office suites, providing a mechanism for easy communication. Even faculty professional development is created to allow both departments equal benefit, by focusing on teaching and learning. Through the symbolical frame, social functions are built around opportunities to celebrate and connect and are well attended by faculty and staff. Loss of departmental identity and faculty/staff participation in these optional "culture-building" activities, and expansion of the College to include the Physician Assistant program have 


\section{American Journal of Pharmaceutical Education 2018; 82 (5) Article 7160.}

been identified as challenges. Being intentional and diverse in efforts to foster a collaborative culture is critical in the development of meaningful traditions, living our mission, and fulfilling our vision.

Fostering Interdepartmental Synergy through Engaging Relationships. Christine R. Birnie, St. John Fisher College, Keith DelMonte, St. John Fisher College, Kathryn Connor, St. John Fisher College, Todd D. Camenisch, St. John Fisher College, Lisa Avery, St. John Fisher College, Elizabeth Sutton Burke, St. John Fisher College. Introduction: The Wegmans School of Pharmacy (WSoP) is comprised of two departments: Pharmaceutical Sciences and Pharmacy Practice and Administration (PPA). With 34 faculty members, 10 in pharmaceutical sciences and 24 in PPA, collaboration includes scholarship, service, teaching, and social activities. Scholarship: Numerous faculty engage in collaborative scholarship, ranging from the stability of compounded products in clinical settings, to the support PPA faculty provide in the area of statistics and epidemiology. As a result, numerous co-authored publications and presentations have resulted from these collaborations. Service: The WSoP has a strong commitment to service to the school, the profession, and the community. All faculty and staff participate in the school's annual Service Day event, and in student organizationsponsored community events throughout the year. Faculty from both departments lead the school's summer medical mission/service trips and mission rotations. Teaching: Recent alignment of the curriculum ensures clinical topics are presented in the basic science courses, prior to being taught in Applied Pathophysiology and Therapeutics. Several elective courses are co-instructed by faculty across the departments and more than half of the pharmaceutical science faculty precept students on APPE rotations. Social: Social, service and team-building activities for the faculty and staff are scheduled throughout the year and built into the school's annual retreat to improve interpersonal and working relationships. Conclusion: The cohesiveness of the departments allows students to gain insight into professional practice and collaboration, and reinforces academic concepts. These collaborations promote a more productive and efficient working environment for faculty amongst both departments.

From 3D Visualization to Human Patient Simulation: Applying Molecular Models to Clinical Practice. Amanda M. Chichester, The University of Rhode Island, Bongsup Cho, The University of Rhode Island, Patrick S. Kelly, The University of Rhode Island, Nickolai K. Morin, The University of Rhode Island, Catherine M. Chatowsky, The University of Rhode Island. Doctor of Pharmacy (PharmD) programs strive to create learning experiences that actively engage learners and address the diverse learning needs of students (ACPE Standards 10.12 and 10.13). We sought to determine pharmacy students' perceptions of the use of 3D visualization tools coupled with a high-fidelity human patient simulation (HPS) exercise within the integrated pharmacy practice laboratory curriculum. Second professional year (P2) PharmD students enrolled in a central nervous system (CNS) course participated in the integrated pharmacy practice laboratory. The 3D visualization activities focused on the structure activity relationships (SAR) of chosen central nervous system drug molecules and the pathway for dopamine synthesis. For the accompanying high-fidelity HPS exercise, students treated a patient who presents with a possible benzodiazepine and ethanol overdose. Student perceptions of the 3D visualization tools coupled with the HPS exercise were assessed through pre- and post- laboratory surveys (1-5 Likert Scale). Prior to attending the laboratory $45 \%$ of students agreed that $3 \mathrm{D}$ visualization tools (i.e. molecular modeling laboratory and 3D printing) should be used within the PharmD curriculum. After attending the laboratory $70 \%$ of students agreed that $3 \mathrm{D}$ visualization tools should be used within the PharmD curriculum. Students identified that incorporation of practice-based experiences with $3 \mathrm{D}$ visualization tools, especially those which demonstrate relevance of challenging concepts such as CNS medicinal chemistry, are valuable additions to the PharmD curriculum. Students' also displayed a strong desire for similar future learning experiences.

From Admissions to Graduation: Integrating Learning to Develop Practice Ready Pharmacists. Bradley M. Wright, Auburn University, Daniel W. Surry, Auburn University, Timothy Moore, Auburn University, Lori B. Hornsby, Auburn University, Julaine M. Fowlin, Auburn University, Channing R. Ford, Auburn University, Karen F. Marlowe, Auburn University. Auburn's Harrison School of Pharmacy (AUHSOP) has revised its curriculum with a vision to create an integrated curriculum through the utilization of backward design focused on the achievement of the AUHSOP ability based outcomes. The faculty were assigned to curricular design and teaching teams. Each team includes faculty from multiple disciplines. Students throughout the revised curriculum learn about drugs and diseases as they master a series of approximately 300 performance based competencies across various learning experiences. Previously basic science content was delivered in the first two years in discipline focused courses. Learning experiences are based on disease states and are designed around the Pharmacists' Patient Care Process. Performance based competencies are interwoven into units to encourage authentic learning and promote retention. Content related to the disease and therapeutics is guided by a predetermined progression guide developed to scaffold learning over three years. The P1 year sets the foundation for an understanding of drugs and diseases as students are exposed to the pathophysiology, mechanism of action and monitoring of medications. The P2 year provides students more depth regarding drugs and diseases as they investigate the relationship between medicinal chemistry, and properties impacting ADME. Students explain why specific drugs are effective for disease states. The P3 year provides deeper understanding of patient specific factors effects on safety, efficacy, drug interactions, pharmacogenomics, and ADME. One year into implementation 39 integrated units have been delivered. Preliminary results will be presented including faculty/student feedback.

From Bench to Bedside to Policy Making: An Interdisciplinary Approach to Teaching Substance Abuse Research. Otito F. Iwuchukwu, Fairleigh Dickinson University, Dongmi Kim, Fairleigh Dickinson University. Substance use disorders (SUDs) have recently been receiving increased attention from policy makers and government sectors thereby opening unprecedented doors to pharmacists, such as in community distribution of naloxone. At the same time, the research of substance dependence continues to be rigorous surrounding a wide array of topics including pharmacogenomics of dependence, effectiveness of therapeutic interventions, prevention education and population health. Pharmacists and scientists cut across many roads from basic science research to patient intervention in various settings of SUDs. In this second-professional year elective course, paradigms of substance abuse research from bench to bedside to policy making are introduced. Current status of SUDs is presented in order, starting with ancient history of illicit substances. Genomic basis of substance dependence that may contribute to the biological and psychological etiology of SUDs and multiple social constructs of substance abuse patterns are discussed. Students role-play and practice Screening-Brief Intervention and Referral to Treatment (SBIRT), debate over marijuana legalization, create and present posters on naloxone use, and discuss clinical scenarios with a substance abuse counselor. Students observe that basic science research does not end 


\section{American Journal of Pharmaceutical Education 2018; 82 (5) Article 7160.}

where patient care begins; rather, that research science and patient intervention are intertwined and build on each other. Student learning climaxes with a public health opportunity to impact our community with a campus outreach event. We believe this unique offering of interdisciplinary, interprofessional learning prepares students to expand on traditional roles of pharmacists and possibly inventing nontraditional roles for pharmacists in the research of SUDs and treatment thereof.

From Laboratory to Clinic and Beyond: Creating Broad Research Opportunities for PharmD and PhD Students. William P. Petros, West Virginia University, Grazyna D. Szklarz, West Virginia University, Mary K. Stamatakis, West Virginia University, Usha Sambamoorthi, West Virginia University, S. Suresh Madhavan, West Virginia University, Paul R. Lockman, West Virginia University, Marina Galvez Peralta, West Virginia University. Translational research is a multidisciplinary endeavor which allows students to benefit from cross-talk between disciplines as a foundation for their clinical or research careers. $\mathrm{PhD}$ students preparing for careers in research can benefit from clinicians to make their research more translationally relevant. PharmD students may be planning to pursue a research path, but also benefit from the development of critical thinking and analytical skills that research experience provides. The WVU School of Pharmacy offers different avenues for PharmD students to explore research: required research courses in Pharmaceutical Sciences and Evidence-Based Medicine; an electives-based, pathway in Translational Research; a structured research mentoring program; experiential opportunities (clinical trials center, pharmaceutical industry, regulatory science). Students can opt to concurrently complete a certificate or master's degree in Clinical and Translational Science. The Pharmaceutical and Pharmacological Sciences $\mathrm{PhD}$ program provides opportunities to be involved in target identification, discovery of novel therapeutic agents, and pre-clinical and clinical pharmacology using cutting-edge technology in fields of nano-science, 3-D printing, pharmacogenomics/ pharmacokinetics, cancer therapeutics, neuroscience, and cardiovascular diseases. The Health Services and Outcomes Research PhD program focuses on improving healthcare and pharmacy practice in West Virginia and nationally by resolving problems related to access, affordability, cost, quality and outcomes of healthcare and developing, implementing, and evaluating innovative services in medication use, community health, preventive care, and chronic disease management. Examples of students involvement in multidisciplinary translational research projects will be provided. Also highlighted will be joint training/educational opportunities between programs.

Growth of Pharmacogenomics at Bernard J. Dunn School of Pharmacy. Timothy J. Bloom, Shenandoah University, Marcia L. Brackbill, Shenandoah University, Katrina Petrov, Shenandoah University, Andy R. Eugene, Shenandoah University. Pharmacogenomics was first introduced into the Bernard J. Dunn School of Pharmacy core curriculum in 2006. Currently, four PharmD courses are taught by faculty from the Biopharmaceutical Sciences, Pharmacogenomics and Pharmacy Practice departments to provide a cohesive delivery of biomedical and pharmacogenomics knowledge with clinical applications to our PharmD students. Our mission is to produce leaders in inter-professional, patient-centered care. Coupling that with the growth of pharmacogenomics as both a discipline and a tool for identifying the best patient care, we identified an urgent need for expanding our pharmacogenomics offerings. As of 2018, over 300 drug-gene pairs have been included as pharmacogenomics biomarkers in drug package labeling. Therefore, we recently began offering an online certificate program aimed at providing general knowledge of pharmacogenomics to practicing healthcare professionals. We also identified a gap related to pharmacogenomics guidelines, between interpreting laboratory genomic results and applying genotype-guided prescribing to patient care and research. Therefore, we are developing an M.S. in Pharmacogenomics and Personalized Medicine degree with courses targeting two main areas. Several courses focus on the laboratory genomics methods, bioinformatics, computational genomics, and quantitative pharmacology used to generate pharmacogenomic data. Other courses focus on how pharmacists, physicians, nurses, physician assistants, and other members of the patient care team use pharmacogenomics information in clinical decision making. Our goal is to use an interdisciplinary approach to prepare clinicians to apply and advance current knowledge of drug-gene interactions to optimize patient dosing, minimize adverse drug reactions, and improve patient care.

Impact of the Pre-Matriculation and Post-Matriculation Academic Performance on NAPLEX Scores. Avadhesh C. Sharma, Philadelphia College of Osteopathic Medicine, Michael J. Lee, Philadelphia College of Osteopathic Medicine, Vishakha Bhave, Philadelphia College of Osteopathic Medicine, Shawn D. Spencer, Philadelphia College of Osteopathic Medicine, Naushad K. Ghilzai, Philadelphia College of Osteopathic Medicine, Harish S. Parihar, Philadelphia College of Osteopathic Medicine, Vicky Mody, Philadelphia College of Osteopathic Medicine. Objective: To assess the impact of pre-matriculation and post-matriculation academic performance on NAPLEX scores. Method: PharmD class of 2016 \& 2017 students' pre-matriculation [overall GPA, science GPA, PCAT (composite, bio, verbal, chemistry, reading and quantitative)], post matriculation grades [high stakes milestone exam (PHAR399G), integrated therapeutics (IT), pharmacokinetics (PK), and pharmacy calculations courses (PCal)] and NAPLEX scaled scores at the PCOM School of Pharmacy were retrospectively collected and analyzed for individual pearson's correlations, and by stepwise automated multivariate regression on selected variables to examine the influence of these principal factors in pre- and post-matriculation performance on NAPLEX scores. Results: A total of 174 graduates from the class of 2016 and 2017 were included in the study. Pearson's correlation matrix identified post-matriculation variables as most correlated with NAPLEX scaled scores whereas pre-matriculation variables were not correlated with NAPLEX scores. Mean NAPLEX scaled score was 89.5 (SD 17.04), mean PCalc score was 85.6 (SD 6.79), mean IT score was $82.5 \%$ (SD 5.13 ) and mean PK score was $81.6 \%$ (SD 6.56). Stepwise multivariate regression analysis indicated that NAPLEX performance has significant prediction dependence on PHAR399G and IT course scores, accounting for $52.3 \%$ (adjusted R2 $=0.51, \mathrm{p}<0.01$ ) of the variance in NAPLEX scaled scores. Pre-matriculation academic performance was not found to be related to NAPLEX scores. Implications: Our results indicated that post-matriculation factors may be better predictors of NAPLEX performance compared to pre-matriculation academic factors.

Improving Comprehension and Communication of Pharmaceutical Theoretical Principles. Teresa E. Donegan, D'Youville College, Shoshanna Zucker, D'Youville College, Vicky Belousova, D'Youville College. Introduction: To enhance the comprehension of pharmaceutical science theories and the communication of the underlying causes of disease states, the first semester of the Professional Development of the Student Pharmacist course was redesigned to increase the integration and application and communication of pharmaceutical principles of biochemistry, anatomy and physiology to patient cases. Design: Using a team-based approach, students were asked to apply theoretical principles taught in their biochemistry, anatomy and physiology courses to two patient cases; both adults, one with hyperlipidemia, and the other with hypertension and chronic obstructive pulmonary 


\section{American Journal of Pharmaceutical Education 2018; 82 (5) Article 7160.}

disease. Teams were asked to describe the principles in layman's terms for both cases and then to tailor the information to the patients in each case either by description or diagram using analogies. Faculty from biochemistry and anatomy and physiology facilitated the debriefing of both case-based exercises. Evaluation and Assessment: A multimethod assessment will be used to assess the effectiveness of learning methods. Students' performance on an Objective Structured Clinical Examination question that asked them to define HDL and LDL will be compared to peers in previous classes. Team performance on tailoring communication of hyperlipidemia and COPD will be analyzed. Student perceptions of the impact of learning activities on comprehension and communication will be assessed through a cross-sectional survey administered through Qualtrics, a secure survey software.

Improving the Comprehension and Communication of Pharmaceutical Theoretical Principles. Teresa E. Donegan, D'Youville College, Shoshanna Zucker, D'Youville College, Victoria Belousova, D'Youville College. Introduction: To enhance the comprehension of pharmaceutical science theories and the communication of the underlying causes of disease states, the first semester of the Professional Development of the Student Pharmacist course was redesigned to increase the integration and application and communication of pharmaceutical principles of biochemistry, anatomy and physiology to patient cases. Design: Using a team-based approach students were asked to apply theoretical principles taught in their biochemistry, anatomy and physiology courses to two patient cases; both adults, one with hyperlipidemia, and the other with hypertension and chronic obstructive pulmonary disease. Teams were asked to describe the principles in layman's terms for both cases and then to tailor the information to the patients in each case either by description or diagram using analogies. Faculty from biochemistry and anatomy and physiology facilitated the debriefing of both case-based exercises. Evaluation and Assessment: A multi-method assessment was used to assess the effectiveness of learning methods. Students' performance on an Objective Structured Clinical Examination (OSCE) question that asked them to define HDL and LDL were compared to peers in previous classes. Team performance on tailoring communication of hyperlipidemia and COPD were analyzed. Student perceptions of the impact of learning activities on comprehension and communication were assessed through Qualtrics secure survey software. Discussion: Students' performance on the OSCE questions were significantly higher compared to peers from previous years. Performance scores on communication activities will be evaluated as compared to established outcomes. The data suggests a benefit in continuation of this model system for the future

Initiatives to Encourage Interdepartmental Collaborations to Support Faculty and Student Development. Anna K. Morin, MCPHS University-Worcester/Manchester, Maryann Cooper, MCPHS University-Worcester/Manchester, Cheryl R. Durand, MCPHS UniversityWorcester/Manchester, Robert Campbell, MCPHS UniversityWorcester/Manchester, Katherine Carey, MCPHS University-Worcester/ Manchester, Paul P. Belliveau, MCPHS University-Worcester/Manchester, Abir Kanaan, MCPHS University-Worcester/Manchester. MCPHS University- School of Pharmacy Worcester/Manchester strategic plan supports interdepartmental collaboration and the development of opportunities to promote educational research and postgraduate education(PGE). Faculty from Pharmacy Practice and Pharmaceutical Sciences collaborate on service, scholarship, and teaching initiatives. The interdepartmental Pharmacy Academic and Leadership Support Committee promotes teaching and research collaborations through professional development activities. Faculty share pedagogical strategies, classroom experiences, and scholarly work. In a required course, Dean's Seminar, faculty introduce students to opportunities for enhancement including research, which may be pursued via a directed study, concentration, or extra-curricular activity. One concentration, Pharmaceutical Cancer Research, provides structured research training in pharmaceutics and/or oncology-related fields of pharmacy. This concentration allows students to develop problem-solving skills and provides deeper insights into theoretical and experimental research topics. Participants complete modules on laboratory research, two elective courses, and an advanced experiential education rotation. The concentration was started in 2016 and has enrolled 46 students. To date, 18 students are currently fulfilling requirements, and 15 students completed all requirements; 8 of these students were successful in obtaining PGE. Furthermore, faculty serve as preceptors for various residency and biopharmaceutical-focused fellowship programs. In 2017, an interdepartmental PGE Preparation Committee implemented a series of sessions to introduce students to residency and fellowship programs, application processes, and strategies for success to enhance students' personal marketability. A mentorship component was also developed and matched students with faculty mentors from either department based on the PGE program of interest. Based on feedback, the series and mentorship program continued in 2018 .

Institute for Advanced Medical Research at Mercer University: Translational and Clinical Research Opportunities for Students. Angelo Sambunaris, Mercer University, Nader H. Moniri, Mercer University. The Mercer University College of Pharmacy has developed a unique collaboration with the Institute for Advanced Medical Research, a private clinical research organization, to afford patientcentered clinical and translational research opportunities to Pharm.D. and other healthcare students. The Institute for Advanced Medical Research at Mercer University conducts Phase II-IV clinical trials at Mercer's Center for Clinical Research. Current studies address a variety ofCNS-centric disease states including depression, anxiety, PTSD, and binge eating. Through this collaboration, Pharm.D. students can engage in clinical research through second- or third- year didactic elective research courses that can span one or both academic semesters, as well as 5-week Advanced Pharmacy Practice Experiences in their fourth year. The collaboration also plays an integral part in training of students within the Pharm.D./Ph.D. Clinical Scientist Program, and provides practice-based experiences with clinical methodologies in investigation of novel pharmacotherapeutics, under the supervision of a medical director and the research team. In the first year of the collaboration, over 30 Pharm.D. students participated in clinical research projects, and this is expected to grow in the subsequent years. Overall, it is anticipated that the collaboration would enhance student respect for, and engagement in, clinical and translational research and provide insight into career opportunities within the pharmaceutical and biotechnology industries, regulatory agencies, as well as clinical research organizations.

Integrated Case Based Learning in Pharmacotherapy Recitation Series. Hershey S. Bell, Lake Erie College of Osteopathic Medicine, Katherine M. Tromp, Lake Erie College of Osteopathic Medicine, Kenneth S. Bauer, Lake Erie College of Osteopathic Medicine, Rahul R. Deshmukh, Lake Erie College of Osteopathic Medicine, Rinita H. Vaishnav, Lake Erie College of Osteopathic Medicine-Bradenton, Nina Pavuluri, Lake Erie College of Osteopathic Medicine, Lana Hochmuth, Lake Erie College of Osteopathic Medicine, Deepak Gupta, Lake Erie College of Osteopathic Medicine. Objective: To evaluate students' perceptions of the redesigned pharmacotherapy recitation course with the inclusion of integrated case-based learning (CBL) activities to promote higher order learning. Methods: The LECOM School of Pharmacy delivers an integrated Pharmacotherapy, Medicinal Chemistry and Pharmacology lecture-based course series. 


\section{American Journal of Pharmaceutical Education 2018; 82 (5) Article 7160.}

Parallel to this is a Pharmacotherapeutics Recitation course that provides active learning opportunities. Integrated CBL activities have been added to the recitation course to promote higher level learning of students. These activities merge material from medicinal chemistry and pharmacology with the pharmacotherapy clinical cases. The goal is to prepare students for clinical practice through the use of integrated clinical cases that link theory to practice, through the application of knowledge using inquiry-based learning methods. A 12-item survey instrument was developed and distributed to students at the LECOM School of Pharmacy to evaluate the students' perceptions and impact of the integrated CBL activities on their higher order thinking. Frequency data was tabulated. Results: Student perceptions of integrated CBL activities were positive. Over $80 \%$ of the students responding strongly agreed or agreed to questions pertaining to the positive impact of the integrated CBL activities on enhanced higher order learning. The P3 students better appreciated the benefits of integration compared to the $\mathrm{P} 4 \mathrm{stu}-$ dents. Implications: Integrated case-based learning allows class-time to be used for higher levels of learning and strengthens students' knowledge and application of foundational sciences in solving clinical cases in pharmacotherapeutics. This format will be further developed to enable retention and application of all foundational sciences in clinical cases.

Integrated Pharmacotherapy: Merging Science and Practice for Transformative Learning. Katherine A. Kelley, The Ohio State University, Justin M. Habash, The Ohio State University, Nicole C. Kwiek, The Ohio State University, Keli Hu, The Ohio State University, PuiKai (Tom) Li, The Ohio State University, Alexa S. Valentino, The Ohio State University, Laura E. Hall, The Ohio State University, Brianne L. Porter, The Ohio State University, Bella H. Mehta, The Ohio State University, Ruth E. Emptage, The Ohio State University, Maria C. Pruchnicki, The Ohio State University. Current standards for pharmacy education emphasize integration of disciplinary content, and literature supports its value in improving health professions education. The Ohio State University College of Pharmacy prioritized integration as part of curricular revisions of the BS Pharmaceutical Sciences (BSPS) and Doctor of Pharmacy (PharmD) curricula. In both, didactic courses were redesigned to connect science-based and practitioner faculty through enhanced problem-based teaching. The Molecules to Medicines (BSPS) and Integrated Pharmacotherapy courses (PharmD) were designed with an integrated framework focusing on molecular, cellular, and pathophysiologic mechanisms of disease, guiding rational drug treatment. Revisions were led by coordinating teams. Faculty addressed integration at three levels: sequence, course, and class session within course. At each level, purpose and intended outcomes were identified. Disciplines included physiology/pathophysiology, pharmacology, medicinal chemistry, pharmaceutics and therapeutics. Using the integration framework of Goldman and colleagues (Academic Medicine, 2012), integration occurred through temporal coordination/correlation (aligning related topics, addressing multiple perspectives) and co-teaching (planning and delivering content through teaching teams). Student assessment occurred during graded patient cases (PharmD) and/or integrated case-based examinations (BSPS and PharmD). Feedback from students in first-time offerings of the courses indicated they perceived benefits in effective learning and improved critical thinking/problems-solving approaches. Students appeared to value the integrated experience. Activities provided College of Pharmacy faculty and students the opportunity to collaborate in new ways, and enhance communication across disciplines. Key elements for successful implementation included faculty buy-in, scheduled team meetings and peer teaching. Additional faculty resources, multi-level oversight and coordination were needed; ongoing quality improvement is planned.
Integrating Pharmaceutical Sciences and Pharmacy Practice Using a Novel Course in Compounding Sterile Preparations. Shanada Monestime, University of North Texas Health Science Center, Theresa Day, University of North Texas Health Science Center, Dan Hooper, University of North Texas Health Sciences Center, Drew Thomas, University of North Texas Health Sciences Center, Randy Martin, University of North Texas Health Science Center. Courses in compounded sterile preparations (CSPs) are an opportunity to highlight the natural intersection of pharmaceutical sciences and pharmacy practice. Sterile compounding pharmacists integrate concepts in medicinal chemistry to exercise professional judgment as it pertains to beyond use dating, preparation formulae development, and answering questions concerning IV compatibility. However, many sterile compounding courses provided by colleges of pharmacy primarily focus on aseptic technique, instead of pharmacist-specific tasks requiring professional judgment. At University of North Texas System College of Pharmacy (UNTSCP), students complete a course in sterile compounding. From 2016 to 2017, our team transformed an existing sterile compounding course to deliver a more comprehensive experience that included equal instruction in technical skills, analytical skills, and true-to-practice simulation. Technical skills included calculations, hand hygiene and garbing, and aseptic technique. Analytical skills included quality evaluation, application of compatibility and stability data, determination of beyond use date (BUD), and synthesis of recommendations for drug administration. The course culminates in a comprehensive OSCE in which students completed a series of activities that actually occur in sterile compounding laboratories and require students to exercise professional judgment. Course assessments demonstrated improvement in student satisfaction and improved student performance in the new course model. Despite the reduction in time spent on aseptic technique to focus on other content areas, competency actually improved. This novel sterile compounding course provides a successful blueprint for instruction and assessment that highlights the integration of science and practice and provides students with true-to-practice experiences which develop their ability to exercise professional judgment.

Integrating the Electronic Health Record into the First Three Years of the Pharmacy Curriculum. Kassandra Lecci, Nebraska Medicine- University of Nebraska Medical Center, Kristen M. Cook, University of Nebraska Medical Center. The electronic health record (EHR) and use of technology have become a vital part of the patient care process. Student pharmacists must be able to use the EHR and apply their knowledge to take care of patients. During the past three years, our College has incorporated the EHR in every year of our didactic curriculum. Students start with exploratory activities during their first year. The second and third year students receive training in both the inpatient and outpatient EHR. Nine cases, with increasing complexity, have been incorporated thus far. Each case is built with the help of a pharmacist who is an Educational Environment Coordinator for our health system's EHR. The process is collaborative between the faculty member and the coordinator. Students perform clinical activities in the EHR including: documentation, order assessment, collection of patient information, and problem solving. They interact in groups, as well as individually. Some cases incorporate principles learned in their pharmaceutical sciences courses. Our infectious disease case requires the student to apply pharmacokinetic principles to a simulated patient and ultimately document their consult in the EHR. A series of oncology cases allow students to evaluate chemotherapy orders and incorporate principles learned in pharmaceutical science courses. We have utilized faculty education and engagement of our clinical faculty to help move this initiative forward. 


\section{American Journal of Pharmaceutical Education 2018; 82 (5) Article 7160.}

The development of an assessment tool for EHR skills is being developed to track student progress through these activities.

\section{Integrating the Foundational Pharmaceutical and Basic Sciences} with Pharmacy Practice through Interconnected Course Sequences. Mitchell R. Emerson, Midwestern University/Glendale, Michael A. Dietrich, Midwestern University/Glendale, Shawn Tennant, Midwestern University/Glendale, Stephanie J. Counts, Midwestern University/ Glendale. The three-calendar year accelerated Doctor of Pharmacy program at Midwestern University College of Pharmacy-Glendale includes an eight-quarter, Professional Skills Development (PSD) progressive course sequence throughout the didactic curriculum. The first-year of PSD meets on a weekly basis and emphasizes active learning techniques including workshops, games, and standardized patient simulations, as well as lecture with guided discussion. The series reinforces integrated application of foundational basic and pharmaceutical scientific knowledge within pharmacy practice. A continuous quality improvement program allows PSD to be fluid considering learning styles and needs of each incoming class. Content pulls from courses in physiology and pharmaceutics, as well as the pharmacotherapeutic core of the curriculum, the Integrated Sequence (IS) which, designed in an organsystem, disease-state based manner, combines pathophysiology, pharmacology, medicinal chemistry, and therapeutics. Beginning in didactic quarter three, the IS runs concurrently with PSD and workshops within PSD reinforce foundational material from IS within infused patient case studies. Examples of crossover activities within PSD include workshops/ guided discussion on endocrine physiology, diuretic pharmacology, microbiology and infectious disease, as well as endocrine, cardiovascular, central nervous system, and infectious disease patient cases. A case-based medicinal chemistry workshop is being developed. At the end of each didactic year, the PSD sequence concludes with an Objective Structured Clinical Exam (OSCE). In particular, after the first year, students must demonstrate competency during a seven-station OSCE that is designed to have $50 \%$ of the competencies from pharmacy practice, including pharmacy law, and $50 \%$ from the basic and pharmaceutical sciences, including calculations and pharmaceutics.

Integrating the Instruction of Medicinal Chemistry and Pharmacology to Improve Student Learning and Inform Therapeutics. Richard W. Dudley, University of Findlay, Ryan A. Schneider, University of Findlay, Lori Ernsthausen, University of Findlay, Jason W. Guy, University of Findlay. Objective: To identify if co-teaching of medicinal chemistry and pharmacology enhances students' performance in an endocrinology course. Methods: Instructors in the medicinal chemistry and pharmacology portions of the endocrinology course at the University of Findlay merged their content together in order to provide a co-teaching experience to students in the course. Faculty presented concepts together during the same class. A survey was sent to students who participated in the co-taught course $(n=49)$ to collect qualitative feedback on the technique. Midterm scores for the 2018 course were compared to 2016 and 2017 data $(n=173)$. The only significant change to the course in 2018 was co-teaching. A t-test was used to compare individual midterm scores to 2018 midterm scores. Alpha for the study was 0.05 . Results: The average exam score in 2016 was $82.4 \%$ and in 2018 the average was $80.3 \%(\mathrm{p}=0.35)$. The average exam score in 2017 was $78 \%$ ( $p=0.34$, compared to 2018). Students performed progressively better each year in the pharmacology category and progressively worse in the medicinal chemistry category, however these differences were not significant $(\mathrm{p}=0.86$ and $\mathrm{p}=0.65$, respectively). Students' performance in the therapeutics section of the assessment was similar between years. Implications: Students performed similarly on the midterm exam in the endocrinology course throughout all three years studied. Thus, co-teaching did not improve or worsen overall performance in the course. Therefore, co-teaching was not shown to be useful to enhance performance, but may be warranted if students prefer this method of instruction.

Integration and Sequencing of Didactic and Active Learning with Electronic Course Management and Testing. Shannon J. Drayton, Medical University of South Carolina, Patrick M. Woster, Medical University of South Carolina, Kelly R. Ragucci, Medical University of South Carolina, David W. Shirley, Medical University of South Carolina, Yuri Karl Peterson, Medical University of South Carolina. Key technologies are maturing at a rapid pace, allowing for increased communication and access to qualitative and quantitative metrics to assess outcomes. As part of our continual modernization process and in response to curricular and assessment needs, we have resequenced course material while completing integration of multiple enterprise software solutions (Moodle, Panopto and ExamSoft) into the first year Pharmacy curriculum. One major key to success is proper sequencing of course material where the appropriate didactic material precedes and supports a simulated learning laboratory. The work we have done to integrate our didactic Dosage Forms and Drug Delivery Systems course with the active Compounding and Applied Pharmaceutics Laboratory is presented. Student satisfaction was highly positive and increased in parallel with student use of the technology and improved student performance. Course objectives and test questions are tagged with CAPE Outcomes and can be used to further optimize, customize and enhance lecturer and student achievement of the outcomes. At our institution, student performance and outcomes have been improved through the integration and sequencing of didactic and active applied laboratory learning supported by technology solutions.

Integration of Disciplines in Teaching of Parkinson's Disease. Kelly M. Shields, Ohio Northern University, Kelly M. Kroustos, Ohio Northern University, Jeffrey J. Christoff, Ohio Northern University, Mark E. Olah, Ohio Northern University. Introduction: At the Raabe College of Pharmacy at Ohio Northern University, the latter academic years consist of therapeutic modules defined by organ systems. Disease states are presented in discrete lectures in a sequence of pharmacology, medicinal chemistry and therapeutics. Subject areas are covered by faculty with expertise in the specific discipline. Methods: For the past three years, an integrated approach among disciplines has been employed for teaching of Parkinson's disease (PD). Instructors for pharmacology, medicinal chemistry and therapeutics join each other simultaneously for lecture to provide "real-time" integration of material. The clinician provides an introduction to $\mathrm{PD}$ with description of patient demographics and presentation. This is followed by presentation of pathology at neuroanatomical and molecular levels with connection to patient presentation. Agents for PD are presented sequentially with the basic science followed by therapeutics. Connections are made between all instructors with frequent reference to material just presented. Results: The integrated teaching of PD as compared to individual instructors presenting subject matter in a continuous session has mixed results. Exam scores are not substantially different between the integrated approach and previously employed presentations. Student evaluations are varied as many note connections by instructors enhance understanding while a substantial number appreciate the segregated approach. Conclusion: The integrated approach to teaching provides an interactive approach among instructors in the teaching of a disease state. We will continue to gather data to assess this methodology in PD and other areas in which this approach has been initiated. 


\section{American Journal of Pharmaceutical Education 2018; 82 (5) Article 7160.}

\begin{abstract}
Integration of Science and Practice, Problem-Solving, Literature Analysis and Scholarship: A Vertical Curricular Thread. Glen L. Stimmel, University of Southern California, Susie H. Park, University of Southern California, Roger F. Duncan, University of Southern California, Aileen C. Li, University of Southern California, Maryann Wu, University of Southern California, Ian S. Haworth, University of Southern California, Rory O'Callaghan Kim, University of Southern California. Objectives: A new curriculum has been implemented at the USC School of Pharmacy over the last three academic years (starting in 2015-16). Objectives include integration of pharmacy relevance and problem-solving into science courses (P1), development of literature analysis and scholarship skills (P2), and completion of a scholarly project with a major component of outcomes research (P3). Method: Introduction of these curricular activities has been monitored through regular assessment, including mid- and end-of-semester surveys of students; meetings among course coordinators; and establishment of a scholarly project committee comprising clinical and basic science faculty. Results: Responses of students to a revised Pharmacogenetics course (P1) showed an increased perception of pharmacy relevance, with scores for this question increasing from an average of $3.5 / 5$ for the last three years of the old curriculum to $4.3 / 5$ for the first three years of the new curriculum. The more recently introduced Literature Analysis (P2) and Scholarly Project (P3) courses still have average scores below $4 / 5$. However, these courses provided the basis for successful completion of $>70$ scholarly projects that have contributed to many conference presentations in the current academic year. Implications: The new curriculum has a strong vertical thread from pharmacy-relevant problem-solving to scholarly activity, which allows students to excel in diverse areas of particular individual interest. We believe that these activities will produce a new generation of Pharm.D. graduates with the critical thinking skills to adapt to a changing professional environment. Challenges remain in showing students the importance and relevance of these skills to their career.
\end{abstract}

Interdepartmental Faculty Collaboration to Validate Antimicrobial Dosing Recommendations for Molecular Adsorbent Recirculating System Dialysis. Erika Aldag, Aurora St Luke's Medical Center, Uvidelio Castillo, Concordia University Wisconsin, Lynne A. Fehrenbacher, Concordia University Wisconsin. Background: MARS ${ }^{\circledR}$ is a liver support system designed to remove hydrophilic compounds and highly protein bound lipophilic substances that accumulate during liver failure. This poses the risk of removal of antimicrobials that are highly bound to albumin. Objective: To evaluate antimicrobial dosing recommendations for MARS ${ }^{\circledR}$ to determine if clinically appropriate serum concentrations are achieved based on the minimum inhibitory concentration (MIC) distribution of historical isolates in a Surgical Intensive Care Unit (SICU). Methods: The project was a collaboration between the departments of Pharmaceutical Sciences and Pharmacy Practice. A pharmacy practice faculty was involved with a project to develop antimicrobial dosing guidelines for MARS ${ }^{\circledR}$ patients at the clinical practice site. To gain confidence in recommendations, an observational study was designed that included serum sampling of MARS ${ }^{\circledR}$ patients. Pharmaceutical Sciences performed serum level analysis of antimicrobial concentrations in a blinded fashion. Pharmacy students were incorporated into both the science and clinical aspects of the project. Results: Six patients were analyzed. Drugs included cefepime, fluconazole, metronidazole, piperacillin/tazobactam $(\mathrm{P} / \mathrm{T})$, and vancomycin. Concentrations were at adequate levels based on SICU MIC data except for piperacillin. Two patients on $\mathrm{P} / \mathrm{T}$ were retrospectively assessed as under-dosed preMARS ${ }^{\circledR}$ and had low concentrations. Increasing P/T dose and extending the infusion during the MARS run in one patient yielded a clinically appropriate post-run P/T level. Conclusions: This interdepartmental faculty collaboration supported clinical practice recommendations with objective laboratory data. The recommendations for empiric antimicrobial dosing in MARS ${ }^{\circledR}$ patients achieved adequate concentrations for the drugs sampled in our small patient population.

Interdisciplinary Approaches to Combating the Opioid Use Disorder Epidemic: Lessons Learned From Howard University. Toyin S. Tofade, Howard University, Mary Maneno, Howard University, Simisola Williams, Howard University, Casey Walker, Howard University, Ginika Ezeude, Howard University, Jayla Briggs, Howard University, Adaku Ofoegbu, Howard University, Earl Ettienne, Howard University. Background: The United States opioid use disorder (OUD) epidemic has been declared a public health emergency. In response to the OUD epidemic, the White House Office of National Drug Control Policy (ONDCP) released a report detailing 56 recommendations to address the epidemic. In keeping with Howard University's motto of "truth and service," a multidisciplinary team representing pharmacy, medicine, dentistry, nursing and allied health, social work, business, law, and divinity hosted an Interdisciplinary Policy Symposium (IPS) addressing the opioid epidemic. Purpose: The purpose of the IPS was to garner knowledge from an interdisciplinary team of experts in order to provide innovative solutions to addressing the opioid epidemic. Methods: Six panels including a pre-symposium panel of deans from the convened schools and colleges discussed the opioid epidemic from an educational, legislative, regulatory, clinical, enforcement, and religious perspective. The panelists provided insight based on their area of expertise and addressed questions from the audience. Results: The findings from the panelist and audience members, as well as information in the literature, complemented and further extended the recommendations presented by the ONDCP. Discussion: In past substance abuse epidemics, unbalanced approaches that overemphasized the role of the criminal justice system and minimized the role of healthcare professionals resulted in unsatisfactory outcomes. The role of the pharmacist as a member of an interdisciplinary team can provide invaluable support in addressing the opioid epidemic. Conclusion: The proceedings from the IPS lend important information to guide policy prescriptions that can significantly impact the present opioid epidemic.

Interdisciplinary Collaboration: Conducting Translational Research while Teaching APPE, PharmD Honors and Graduate Students. Iman Zaghloul, MCPHS University-Boston, Mark Bohlke, MCPHS University-Boston, Michelle Ceresia, MCPHS University-Boston. The MCPHS University (MCPHSU) School of Pharmacy-Boston is committed to maintaining a multidisciplinary environment conducive to excellence in teaching, scholarship and professional advancement. The objective of this poster is to demonstrate the interrelationship between faculty in the MCPHSU School of Pharmacy and the Tufts University Cummings School of Veterinary Medicine for the purpose of improving animal care through teaching and research. An MCPHSU Pharmacy Practice faculty member serves on an interdisciplinary Veterinary Medicine team to provide care at the Hospital for Large Animals. Together with Pharmaceutical Science faculty members, the team conducts pharmacokinetic and metabolism research to address complex questions in veterinary practice. The collaboration provides a unique opportunity for pharmacy (APPE, Honors, Graduate) and veterinary medicine students to participate in research in the laboratory and clinical setting. Students gain knowledge and skills in evidencebased literature review, study protocol design, sample collection, data analysis, and manuscript preparation. Additionally, pharmacy faculty 


\section{American Journal of Pharmaceutical Education 2018; 82 (5) Article 7160.}

collaborate to develop and enhance the analytical and presentation skills of the APPE students. Students learn to analyze large animal basic science pharmacokinetic studies and present their findings to veterinary residents and faculty at the veterinary school during formal journal club. Didactic lectures on pharmacokinetics have also been presented to the veterinary medicine house staff. The poster will provide an overview of the projects underway and how the collaboration supports professional development for the faculty and educational outcomes for students.

Interdisciplinary Collaboration: Implementation of Pharmacogenomics Education Across the Doctor of Pharmacy Curriculum. Landry Kamdem, Harding University, Gabriella Douglass, Harding University, Scott Weston, Harding University, Julie C. Kissack, Harding University, Sarah E. Griffin, Harding University. Faculty members from the Pharmaceutical Sciences (PS) and Pharmacy Practice (PP) departments at the Harding University College of Pharmacy (HUCOP) have worked collaboratively to implement pharmacogenomics education across the Doctor of Pharmacy curriculum. Individuals from each group teach and conduct research in this emerging interdisciplinary area. Foundational instruction in basic research design is given to pharmacy students in the Biostatistics and Literature Evaluation course during the $\mathrm{P} 1$ year. Pharmacogenomic information for specific drug targets and pharmaceutical agents is included with the appropriate disease states and drug classes throughout the Pharmacotherapy and Pharmacology/Medicinal Chemistry course sequences. In addition, during the $\mathrm{P} 2$ and $\mathrm{P} 3$ years, focused research elective courses are offered that teach students to collect and assess pharmacogenomics data. These courses are tightly integrated with fourth-year APPEs in which students develop and implement a pharmacogenomic-tailored patient care plan in a clinical setting. Closing the loop, students work with residents to contribute to research publications that integrate the use of pharmacogenomic data for patient care into the literature and provide further support for patient services within the state. This approach models the Pharmacists Patient Care Process (PPCP) and provides students with a realistic picture of pharmaceutical science foundational concepts applied to clinical pharmacy services in predominantly rural health settings. In summary, we have intentionally designed and implemented an interdisciplinary, integrated approach to pharmacogenomics education which combines didactic and experiential approaches in our Doctor of Pharmacy curriculum.

Interdisciplinary Formative Assessments to Foster Student Learning in the Dual-Campus Large Classroom Setting. Aimee F. Strang, Albany College of Pharmacy and Health Sciences, Abby M. Boire, Albany College of Pharmacy and Health Sciences, Loraine Silvestro, Albany College of Pharmacy and Health Sciences, Katherine P. Cabral, Albany College of Pharmacy and Health Sciences, Monique R. Bidell, Albany College of Pharmacy and Health Sciences, Gina Garrison, Albany College of Pharmacy and Health Sciences. Introduction: Albany College of Pharmacy and Health Sciences Doctor of Pharmacy P2 and P3 students take an integrated pharmacology/medicinal chemistry/therapeutic (PTPM) course sequence. These courses involved theme-based teaching by Pharmacy Practice (PP) and Pharmaceutical Sciences (PS) faculty. Three PTPM courses utilized a formative assessment method to pair PP and PS faculty in interdisciplinary sessions. Methods: The model was piloted in 2016 then implemented in three 2017-2018 PTPM courses. Course added a class session before each exam where students took a formative "pre-tests" consisting of 10-15 multiple-choice questions prior to PP and PS faculty leading an interdisciplinary exam review session. A brief student survey was issued following the Fall 2016 pilot. A plan was developed to collect student and faculty feedback and assess the impact on student performance. Results: Implementation of pre-test class sessions provided opportunities for students to observe the importance of and practice the skill of integrating knowledge across disciplines. Student performance improved from pre-test to exam in all courses. Comparison of aggregate exam performance compared to classes prior to the intervention were mixed. Student feedback (response rate 80\%; 182/228) from the 2016 pilot was highly favorable. Nearly $80 \%$ of students (143/182) indicated "strongly agree" to the statement that pre-tests were helpful to their learning. Data are pending for ongoing courses. Implications: Use of a formative pre-test prior to exams can create a structured opportunity to integrate PP and PS faculty in a large classroom setting. Preliminary student feedback to this interdisciplinary approach to pre-exam review was highly favorable.

Joint Approaches in Pharmacoepidemiology, Precision Medicine and Pharmacometrics to Explain, Predict and Optimize Drug Therapy. Stephan Schmidt, University of Florida. Clinical translational sciences have been emphasized as interdisciplinary approaches to connect bench and bedside for more than a decade, but truly integrative approaches are still the exception. At the University of Florida, research teams in three areas of excellence, Pharmacoepidemiology, Precision Medicine, and Pharmacometrics \& Systems Pharmacology, have devoted the past two years to identify opportunities for synergistic research and to develop joint approaches. Opportunities appear abundant in an era where accelerated drug approval decisions require innovative approaches to supplement traditional clinical trials and as demand for post-marketing evaluations, risk mitigation, and tailored therapeutic optimization increase. Rather than employing isolated sequential approaches along the drug approval phases, UF's joint program promotes: 1) reciprocal validation approaches where real-world findings regarding drug outcomes are compared to mechanistic models and vice versa; 2) enhancement of designs of both mechanistic and pharmacoepidemiologic studies by integrating findings from one into the design of the other; 3) extrapolation of mechanistic and clinical trial findings regarding effect modification (e.g., drug effectiveness or safety may vary for specific formulations, in select populations or over specific time) to calibrate value of pharmaceutical and other medical technology; 4) advancement of precision medicine and clinical decision-support by integrating epidemiologic, clinical, genomic and metabolomic determinants in prediction modeling. We will present examples of joint programs including a genomic and PKPD-based voriconazole dosing model, a PKPD and outcomes-based comparative safety study of new oral anticoagulants, and use of real-world data to optimize pharmacogenetics-guided therapy.

Micro-credentials and Masters: Engaging Pharmacy Students in Research Projects. Joseph P. Balthasar, University at Buffalo, The State University of New York, Qing Ma, University at Buffalo, The State University of New York, Peter M. Brody, University at Buffalo, The State University of New York, Murali Ramanathan, University at Buffalo, The State University of New York, Calvin J. Meaney, University at Buffalo, The State University of New York, Brian T. Tsuji, University at Buffalo, The State University of New York, Kathleen M. Boje, University at Buffalo, The State University of New York. Our pharmacy students have several options to engage in research activities. The Clinical Pharmacy Research micro-credential program provides pharmacy students with clinical research skills and knowledge in clinical or clinical translational research. The micro-credential program can be completed within 2-3 professional years, and requires elective research courses and non-credit bearing co-curricular activities (ie, development and defending a research protocol, submission of 


\section{American Journal of Pharmaceutical Education 2018; 82 (5) Article 7160.}

a research report, public presentation and defense of the research work, and a poster presentation). A Clinical Pharmacy Research microcredential is noted on the student's transcript. Those students who desire a more immersive experience may elect to pursue the " $4+1$ " PharmD-Masters or " $4+3$ " PharmD-PhD dual degree programs where two degrees are earned. Students in good academic standing must apply and be accepted into the program of their choice. Based on research interests, students are matched with faculty advisors, from a roster of 10 Pharmacy and 14 Pharmaceutical Sciences faculty. Approximately 12-15 pharmacy students every academic year are accepted into one of the research programs. Currently, there are 33 Clinical Pharmacy Research students (in the P2-P4 years), 4 PharmD-Masters students and 1 PharmD-PhD student. Historically, over 35 PharmD graduates earned the Clinical Pharmacy Research notation, 8 students were awarded the PharmD-Masters dual degree, and 1 student was awarded the PharmD-PhD dual degree.

Overview of Structured Co-curricular Plan at St John's University College of Pharmacy \& Health Sciences. S. William Zito, St. John's University, Andrea Watson, St. John's University, Jeanette C. Perron, St. John's University, Diana J. Patino, St. John's University, Cathleen Murphy, St. John's University, Nissa Mazzola, St. John's University, Maria M. Mantione, St. John's University, William M. Maidhof, St. John's University, Louise Lee, St. John's University, Lisa Hochstein, St. John's University, Monica Hwang, St. John's University, Olga Hilas, St. John's University, Laura M. Gianni, St. John's University, Sue Marie Ford, St. John's University, Danielle C. Ezzo, St. John's University, Joseph V. Etzel, St. John's University, John M. Conry, St. John's University, Jennifer G. Chiu, St. John's University, Christine Chim, St. John's University, Judith L. Beizer, St. John's University, Joseph M. Brocavich, St. John's University, Emily M. Ambizas, St. John's University, Jagannath M. Muzumdar, St. John's University. Standards 2016 by the Accreditation Council for Pharmacy Education mandated the incorporation of co-curricular activities: experiences that complement, augment, and/or advance the formal didactic and experiential curriculum. In response to this charge, St John's University College of Pharmacy and Health Sciences developed a structured co-curricular plan for all PharmD students. Implemented in Fall 2017, this plan was developed to stimulate students to participate in self-directed and life-long learning through professional development requirements. During the first month of the academic year, the College's leadership conducted a Town Hall meeting for each class to review the co-curriculum requirements for that year, the available inventory of Co-curriculum opportunities and all deadlines with the students. Co-curricular requirements (a mix of required activities and additional opportunities where students have choices to select activities that are aligned with their interests) will vary by year to coincide with the changing curricular topics. All Co-curricular activities are approved by an ad hoc committee of faculty members led by the Director of Co-curricular events and are linked to established programmatic outcomes. Following completion of each activity the student completes an assessment activity in his or her e-portfolio. All eportfolios will be reviewed before the end of the academic year. Students are required to demonstrate growth and achievement of all Co-curricular outcomes across the program. This plan serves as a co-curriculum model that promotes activities and experiences for student pharmacists. As a College, we will continue to refine this plan to enhance student learning and develop competent healthcare professionals.

Patient Care Project: Connecting Basic Science to the Care of a Patient. Karissa Y. Kim, University of Cincinnati, Bradley E. Hein, University of Cincinnati, Andrea L. Wall, University of Cincinnati, Bethanne Brown, University of Cincinnati. Objectives: To describe the longitudinal Patient Care Project (PCP), a multi-faceted, collaborative approach designed to promote the student's ability to connect the pharmaceutical sciences to pharmacy practice. Design: Beginning in 2000, faculty from both basic science and pharmacy practice created and implemented an integrated Patient Care Project. As part of a 2012 curricular redesign, the PCP was expanded from 1 to 3 years. With expansion, 23 assignments were created/modified ( 8 basic science and 15 practice-based). Year 1 retained the key core elements from the original project by focusing 15 assignments (housed within 9 courses) on one disease/drug combination, culminating in a professional poster presentation. For the remaining 2 years, 8 additional assignments linked 8 science and practice courses. The PCP scope broadened to include a comprehensive review of all the chosen patient's drugs and diseases. This work also culminated in a professional poster presentation designed around the tenets of medication therapy management (MTM). Assessment: All stakeholders are engaged in order to continually improve the PCP. In addition to grades on the individual assignments and poster presentations, students are asked to complete surveys on the perceived achievement of the PCP learning objectives and guided written reflections at years 1 and 3 . Involved faculty are interviewed each fall to determine changes to be made based on previous years' experience with the individual course assignments. Starting in 2017, an Institutional Review Board approved research project will collect patient input on the process at baseline, after year 1 and year 3 .

Pharmacogenetics: A “Crossing-Over" of Academic Departments in a College of Pharmacy. Stephen J. Cutler, University of South Carolina, Kim E. Creek, University of South Carolina, Patricia H. Fabel, University of South Carolina, Michael Shtutman, University of South Carolina, Michael D. Wyatt, University of South Carolina, Whitney D. Maxwell, University of South Carolina. Inspired by a common interest in pharmacogenomics and pharmacogenetics (PGx), faculty in the Drug Discovery and Biomedical Sciences and Clinical Pharmacy and Outcomes Sciences departments within the University of South Carolina College of Pharmacy collaborated to create a PGx elective course. The course provides students with PGx fundamentals and application to develop PGx-driven clinical decision-making. It involves interdisciplinary speakers including a genetic counselor and a PGx medical science liaison. These and other exposures enable students to explore PGx-related career path options. Other course topics include opportunities for PGx-based entrepreneurship, research, and clinical practice. Student outcomes from the course were positive, with most students indicating improved confidence across Genetics/Genomics Competency Center (G2C2) competencies for pharmacists. The college-wide impacts of this collaboration led to a new PGx Advanced Pharmacy Practice Experience and a collegewide Task Force to examine PGx content and delivery across the PharmD curriculum. The goal of the Task Force was to ensure that graduates are well-prepared to make PGx-oriented patient-care decisions. PGx coverage was analyzed within the curricular map. Additionally, faculty were surveyed to assess utilization of PGx in teaching, research, and clinical practice. Opportunities were identified for improved coordination between courses and across the curriculum. A group of faculty who identify themselves as "regularly or extensively" involved with PGx principles, research, or practice was also identified. This group will provide a foundation for future collaborations. Outcomes of the Task Force included recommendations for PGx-oriented faculty development opportunities to optimize PGx training for students.

Pharmacometabolomics, Natural Language Processing and Data Visualization - Potential Applications in Precision Pharmacy Education. Krista L. Donohoe, Virginia Commonwealth University, 


\section{American Journal of Pharmaceutical Education 2018; 82 (5) Article 7160.}

Kacie Powers, Virginia Commonwealth University, Elvin T. Price, Virginia Commonwealth University, Joseph L. McClay, Virginia Commonwealth University, Dayanjan Wijesinghe, Virginia Commonwealth University. Rapid technological advances are fast enabling the personalization of medicine and the approach to pharmacy education must adapt to include these technologies. A required 1-credit, introductory class in personalized medicine has been offered for the past eight years. To better train pharmacy students who intend to pursue personalized medicine as a career, we created a 2-credit, advanced elective on personalized medicine in fall 2017. The elective introduced the concepts of pharmacometabolomics, with an emphasis on the importance of understanding the effects of one or more pharmaceutical agents on the overall metabolism of the body at an individual level. However, understanding the relevance of such changes with respect to possible complications often requires an exhaustive literature search, a task that is becoming impossible due to the sheer volume of scientific literature. Thus, the topic of literature based discovery (LBD) approaches via automated natural language processing was included in the elective. Output from LBD approaches is best visualized as networks, where the relationships among the identified information are easily comprehensible. Augmented reality was used to demonstrate how to visualize complex information networks in an immersive, interactive and explorable environment. The advanced personalized medicine elective combined these three technologies to enable students to obtain a comprehensive understanding of the metabolic perturbations resulting from an individual's response to a drug regimen. The elective course was positively received by the students indicating their deep interest in maturing technologies for precision medicine.

Pilot of Integrated Pharmaceutical and Clinical Sciences Elective Course to Prepare for Curriculum Transformation. Sharon L. Youmans, University of California, San Francisco, Scott Myers, University of California, San Francisco, Patricia C. Babbitt, University of California, San Francisco, Francesca Aweeka, University of California, San Francisco, Susan M. Miller, University of California, San Francisco, Marcus Ferrone, University of California, San Francisco. The UCSF School of Pharmacy is undergoing curriculum transformation with a new PharmD degree curriculum to launch in July 2018. A key feature of the new curriculum will require teaching students to embrace an "inquiry habit of mind" as they access and incorporate new types of knowledge that typify the rapidly changing landscape of science discovery and apply that new knowledge to contemporary clinical practice. In advance of the new curriculum, the school initiated a series of electives entitled "Seminars in Emerging Science and Practice in Therapeutics" to explore the challenges and develop feasible solutions when faced with merging the basic and clinical sciences in the classroom. These courses were designed to expose students to a diversity of scientific perspectives, drawing from six domains of science. Students from all of the health professions programs were eligible to enroll in the courses. Faculty were selected both within UCSF and industry who lead cutting edge research relevant to disease and therapeutic topics and students participated in facilitated small group discussions to synthesize and integrate aspects of the six domains of science. The electives encouraged students to identify the strengths and weaknesses of health care, where further research is needed, and to think critically about translational applications of the research. Student evaluations of the courses were both positive and frank with respect to their impressions. The SOP has taken this experience and developed subsequent guidelines for course directors responsible for designing the inquiry and discovery project curriculum.

Practice \& Science: Change Creating Collaboration. W. Mark Moore, Campbell University, D. Byron May, Campbell University,
David H. Eagerton, Campbell University, Connie L. Barnes, Campbell University. CPHS has faced significant change in leadership and faculty with a new University President, Dean, Pharmaceutical Sciences (PS) chair and 6 new PS faculty over the past 3 years. Simultaneously, we designed and implemented an integrated curriculum, co-curriculum and assessment plan. New opportunities for collaboration between the PS and Pharmacy Practice (PP) Departments resulted. Integrated Pharmacotherapy (IP) teams of PP and PS faculty were established to develop and teach the new curricular model. Synergistic teams served as a stimulus to identify opportunities and create a better understanding of the roles and responsibilities of each faculty member. These interactions have allowed us to establish new avenues of collaboration, communication, and curricular design and delivery. Outcomes of our collaborations include: 1. Experiential Shadow Program: PP faculty provide shadow experiences at the clinical site to demonstrate the integration of foundational science and clinical knowledge in the practice setting. PS faculty members and CPHS professional staff engage in opportunities to increase their knowledge of pharmacy education, pharmacy practice, and the Campbell Experience. 2. Research Practice Partnerships where PP/PS faculty have actively pursued areas of common interest and expertise to conduct team-based research and scholarship. 3. The development of science content surveys by PS faculty for PP faculty to identify the foundational science concepts that directly apply to their clinical practice. Collaboration yields success. The PS and PP Departments are partnering in new and productive ways to enhance student learning, scholarship opportunities, and faculty satisfaction.

Promoting Student Research in a Three-Year Pharmacy Program. Jacob R. Dunbar, South College. Opportunities for students to gain extensive research experience can be a particular challenge with time and scheduling demands of a 3-year, accelerated pharmacy program. The Concentration in Pharmaceutical Sciences and Pharmacy Research (CPSPR) at South College School of Pharmacy was developed to offer motivated pharmacy students an opportunity to pursue research interests, gain hands-on research experience, and earn recognition for excellence in research which will be a competitive advantage for future career and post-graduate educational opportunities. Student participation in the CPSPR provides the qualified student, research experience to a depth and breadth not otherwise available to all students. Our first CPSR students (Class of 2020) were accepted in the third quarter of their P1 (January 2018) year based upon consideration of GPA (3.0 minimum and no course grade $<$ B), their written statement of interest, and support of the proposed faculty research mentor. CPSPR students must maintain a GPA of 3.0 each quarter, and earn not less than a B in any course taken as a component of the CPSPR. Failure to maintain this standard requires the student to withdraw from the CPSPR. Minimum requirements for successful completion of the Concentration are 18 credit hours of approved research, and researchrelated electives including a research APPE, for which the student must prepare and submit a research manuscript to a peer-reviewed journal for publication. Graduating students who successfully complete the CPSPR will be formally recognized by presentation of honor cords and an award signifying "Excellence in Pharmaceutical Sciences and Pharmacy Research."

Purposeful Collaborations between Practice and Science Faculty Advance Pharmacy Education. David F. Maize, University of the Incarnate Word, Raghunandan Yendapally, University of the Incarnate Word, Elizabeth M. Urteaga, University of the Incarnate Word, Helen E. Smith, University of the Incarnate Word, Sushma Ramsinghani, University of the Incarnate Word, Cheryl K. Horlen, University of the Incarnate Word, Christina Guerra, University of the Incarnate Word, 


\section{American Journal of Pharmaceutical Education 2018; 82 (5) Article 7160.}

Adeola O. Coker, University of the Incarnate Word, Russell T. Attridge, University of the Incarnate Word. The spirit of collaboration at Feik School of Pharmacy (FSOP) has been present since our school opened in 2006. Pharmacy practice and pharmaceutical sciences faculty offices are intermixed on the same floor, promoting interaction, collaboration, and collegiality. Our curriculum includes pharmacotherapeutics modules that integrate practice and science by combining medicinal chemistry, pharmacology, and therapeutics. We work together to develop and facilitate patient cases that have been incorporated into several of our first-year science courses, pharmacotherapeutics modules, and objective structured clinical examinations (OSCEs). In addition, practice and science faculty collaboratively manage an international advanced pharmacy practice experience (APPE) and a "Basic Drug Knowledge and Calculations" program that runs through our Applied Pharmacy Care laboratory series. Integration of faculty in coursework has fostered collaboration in scholarship, service, and professional activities as well. Many of our scholarly partnerships that resulted in poster presentations, podium presentations, and peer-reviewed publications started with ideas generated after practice and science faculty taught together and shared experiences. The FSOP environment cultivates teamwork among practice and science faculty to advance professional and service activities, including committee work, co-advising student organizations, and facilitating development activities for students, staff, and faculty. Collaboration between practice and science faculty is a foundational part of FSOP. Our joint efforts model the School's dedication to training pharmacists skilled at working in teams to improve patient care.

Regis Pharmaceutical Sciences and Pharmacy Practice Faculty: Men and Women in Service of Our Students. Marta J. Brooks, Regis University, Matthew G. Fete, Regis University. The Regis School of Pharmacy's mission is to graduate knowledgeable, skilled and principled pharmacists to become health care leaders in service of others. This is accomplished by delivering a didactic curriculum that integrates the pharmaceutical sciences with pharmacy practice. The school also offers interdepartmental electives, research projects, and service opportunities and two dual MS degrees. The curriculum integrates the basic sciences with pharmacy practice in a pharmacotherapy sequence that includes 12 courses over three didactic years. Students also learn in integrated laboratory courses that span three years and faculty teach formulation in combination with patient care and PPCP. In addition, practice and science faculty collaborate to offer electives (eg, drugs of abuse, immunology concepts, and complementary medicine) and also offer interdepartmental APPEs. Interdepartmental faculty work on research projects which include bench research, the scholarship of teaching and learning, and research in public health. Published examples include interdepartmental articles and presentations on the analysis of over the counter supplements, teaching pedagogy as it relates to team based learning, and the evaluation of immunization rates in our local communities. The ultimate goal of interdepartmental research is to enhance our students' scholarship opportunities. Practice and science faculty collaborate in all aspects of our student's academic and professional growth. Our faculty focuses on the development of the whole pharmacist in order that they are able to apply an outstanding knowledge base, practiced skills, and a principled approach to their service of patients from diverse backgrounds with a variety of different health care needs.

Research Scholars - A Research Concentration Track in PharmD Curriculum to Encourage Research and Innovation. Richard L. Lucarotti, Wayne State University, Lynette R. Moser, Wayne State University, Andrew Berti, Wayne State University, David K. Pitts,
Wayne State University, Minakshi Lahiri, Wayne State University. Wayne State University implemented a renewed PharmD curriculum beginning with the Class of 2020. One component of the renewed curriculum includes a Research Scholars track that aims to provide students authentic hands-on learning and research experience to enhance skills and knowledge in basic and clinical sciences. PharmD students with interest in research are encouraged to apply to the Research Scholars track after the Fall P1 semester. Ten students are offered admission to the program each academic year following a competitive selection process. Scholars enroll in a sequence of coursework electives complementing their longitudinal multi-semester project and are supervised by faculty mentors. They receive valuable experience in research design and analysis, scientific methods, specific laboratory skills, and responsible conduct of research and ethics within areas of pharmaceutical sciences, clinical/translational research, practicebased research and health outcome research. Students have the option to choose from a wide range of research specialties based on their research and professional interests. The research track is structured to promote the students' publication and presentation skills while working with their mentors on research projects. The research experience gained within the track is expected to help these students gain advantage in securing post-graduate residencies, fellowships and advanced graduate degree studies. Students completing the research track will obtain a concentration in research coded on their university transcript at graduation. The application and selection process, research-track curriculum, longitudinal assessment and monitoring mechanisms, and reflections on the 2017 roll-out of the Research Scholars program will be shared in the poster.

Strategically Integrating Biopharmaceutical and Clinical Sciences in Embedded IPE ${ }^{3}$ Program in Doctor of Pharmacy Curriculum. George E. MacKinnon, Medical College of Wisconsin, Beth Krippendorf, Medical College of Wisconsin, Nathan H. Lamberton, Medical College of Wisconsin, Abir T. El-Alfy, Medical College of Wisconsin, Stefanie A. George, Medical College of Wisconsin, Rachel J. Kavanaugh, Medical College of Wisconsin, Karen J. MacKinnon, Medical College of Wisconsin, Susan M. Korek, Medical College of Wisconsin. Future pharmacists must be prepared to work on patient-centered healthcare teams. The new School of Pharmacy at the Medical College of Wisconsin (MCW) places a significant focus on interprofessional education (IPE) in the Doctor of Pharmacy program. This IPE program, called IPE ${ }^{3}$, emphasizes Education, Experiences and Exposures and is longitudinally embedded throughout the 3-year curriculum. These IPE sessions are delivered during (1) foundational Education in classroom and lab sessions, (2) simulations and standardized patient Experiences, and (3) pharmacy practice rotations and patient care labs (Exposures). IPE sessions are embedded throughout the curriculum in the clinical science courses, biopharmaceutical science courses, and Introductory Pharmacy Practice Experiences (IPPE). To track longitudinal IPE progress, students are evaluated using a 20-question pre/post retrospective assessment called Interprofessional Collaborative Competency Attainment Survey (ICCAS). ${ }^{1}$ ICCAS is categorized by the Interprofessional Education Collaborative (IPEC) core competency domains including (1) roles/responsibilities, (2) interprofessional collaboration, (3) values/ethics, and (4) teams/teamwork. ${ }^{2} \mathrm{MCW}$ is currently in the process of first-year IPE program data collection. In addition to the clinical applications in IPPE, integrating biopharmaceutical and clinical sciences in a variety of IPE sessions added value to students' educational outcomes and reinforcement of the basic sciences foundational knowledge. This approach emphasizes the integrated delivery of the didactic and experiential elements of the curriculum. A future IPE session includes the integrated sequence course topic of advanced cardiac life support. The 


\section{American Journal of Pharmaceutical Education 2018; 82 (5) Article 7160.}

embedding of IPE sessions is instrumental in continuing the collaboration across professions and between institutions ultimately aiding in connecting biopharmaceutical sciences with pharmacy practice.

Student Research Influencing College Culture. Debbie C. Byrd, East Tennessee State University, Adam C. Welch, East Tennessee State University, Ralph A. Lugo, East Tennessee State University, Victoria E. Palau, East Tennessee State University, David L. Hurley, East Tennessee State University, David S. Roane, East Tennessee State University. From the initial enrollment of students in the Bill Gatton College of Pharmacy in 2007, a deliberate emphasis was made to encourage PharmD student participation in faculty-mentored research. Engaging students in research has been an organically evolving, administratively supported initiative with up to 60 of our 300 students registered in research electives per semester. Students' enrollment, enthusiasm and achievement led to the creation of a "Concentration in Pharmacy Research (CPRx)." The CPRx requires 12 credit hours of research electives including a capstone project and has had over 60 graduates to date. Completion of the CPRx results in an addendum to the students' diplomas, transcripts and recognition at commencement. Financial support has been provided by college and competitively allocated university funds. For the years 2014 to 2016, peer-reviewed publications contained 42 student authors with 13 students as first authors. There were 144 student authors on abstracts of papers and posters presented at scientific and professional venues. As the program has evolved, student research has become increasingly interdepartmental, involving faculty from both the sciences and practice. This fact suggests that it would be useful to identify facets of the student research program that buttress a desired culture of the college that includes: strengthened student/faculty relationships, improved interdepartmental collegiality, enhanced sense of accomplishment, sense of shared mission, and engagement in critical thinking, active learning and discovery. Analysis of the CPRx program will be presented in the context of a formal definition of college culture that will include metrics of student success and endorsements.

Teaching Students How to Integrate Basic and Clinical Sciences to Enhance Clinical Decision Making. Mustapha A. Beleh, University of Michigan. Purpose: Intentional, collaborative teaching designed to provide students an opportunity to integrate clinical and basic science concepts in clinical decision making prior to starting their advanced pharmacy practice experiences. Methods: Third-year students are required to attend eight two-hour capstone sessions, each focusing on a specific therapeutic area. Sessions are run by experts from each of the three contributing disciplines: Clinical Sciences, Medicinal Chemistry, and Pharmacology. Three to five cases focusing on the session's therapeutic area are discussed during each session. Teams composed of 5 to 6 students discuss each case, define a therapeutic plan and address a number of questions aimed at teaching students how to integrate knowledge from the three disciplines in the context of clinical decision making. One team is randomly selected to present their answers and a class discussion facilitated by the faculty ensues. Assessments: The capstone sessions are graded on a pass/fail basis, with incentives to improve their grade in the course where the sessions are conducted. For each session, students are given an individual assessment to test their preparation and receive a grade based on their participation in the discussions. A final comprehensive exam is administered at the end of the sessions. Future Directions: Based on the first-year experience and student feedback, we plan on modifying some of the cases, adjusting the length of time given to each therapeutic area and providing students with additional resources to prepare for the sessions. Overall, the sessions were well received by students.
Team Science at Skaggs School of Pharmacy and Pharmaceutical Sciences, UC San Diego. Pieter C. Dorrestein, University of California, San Diego, Joseph D. Ma, University of California, San Diego, Jeremiah Momper, University of California, San Diego, Alan Jarmusch, University of California, San Diego, Shirley M. Tsunoda, University of California, San Diego. The Skaggs School of Pharmacy and Pharmaceutical Sciences (SSPPS) at UC San Diego is rooted in the foundational sciences and innovative practice to impact human health. Our vision is to be a catalyst for interdisciplinary collaborations across the school, the medical school, the university, and with local and national biotechnology companies. We highlight one collaborative partnership between the newly formed Divisions of Pharmaceutical Chemistry, Pharmaceutical Sciences and Clinical Pharmacy that exemplifies interdisciplinary collaborations in the area of precision medicine. The goal of precision medicine is to individualize therapy by understanding the genetic and environmental factors that influence efficacy and toxicity. Utilizing our respective strengths across divisions of the school regarding clinical inquiry and ultra-sensitive mass spectrometry technology and metabolomics, we are investigating novel methods for detecting drugs in the skin. Molecular networking allows us to identify chemicals, endogenous compounds, and microbiota including some drugs and metabolites that have been ingested by patients. Clinically, we may be able to harness this technology to monitor drugs and metabolites for therapeutic efficacy/toxicity, adherence, or other biomarkers for disease. We are also investigating how the microbiome affects drug metabolism in humans. Using a phenotyping drug cocktail to evaluate drug metabolizing enzyme activity and perturbation of the microbiome with antibiotic therapy, we hope to elucidate how the gut microbiome influences bioavailability and intestinal metabolism. Evaluating the microbiome as a source of variability will aid in drug optimization. Together we are harnessing our expertise to answer clinically important questions in a deeper and more mechanistic way.

The Frontier of Health Equity: Pharmacy at the Intersection of Precision Medicine and Population Health. Olihe N. Okoro, University of Minnesota, Jacob T. Brown, University of Minnesota, Pamala A. Jacobson, University of Minnesota, Oscar W. Garza, University of Minnesota. Background: Precision medicine is emerging as a means of optimizing drug therapy. Most studies supporting actionable genomic markers have been conducted in Caucasian populations. Genetic diversity in markers related to drugs across global populations is quite profound and important to recognize in pharmaceutical sciences research and implementation into practice. Bridging the gaps between these two areas is critical to advancing the profession and achieving health equity. Objective: To explore how innovative research and education initiatives in two emerging, interdisciplinary areas can help achieve precision treatment, reduce the global burden of disease, and improve health equity. Process: Recent curricular reform, strategic faculty recruitment and development, research investment in the areas of precision medicine and population health, and focusing on medically underserved, rural, and indigenous communities have presented important opportunities for the development of a health equity education framework within the professional and graduate programs in the College. Population health and pharmacogenomics content are intentionally integrated across the core curriculum. A variety of electives have also been developed. Our Institute of Personalized Medicine has undertaken several initiatives focused on defining markers in minority populations, hosts an annual summit on rural/ minority health and precision medicine; and has encouraged interdisciplinary collaborations. Implications: An increased awareness and 


\section{American Journal of Pharmaceutical Education 2018; 82 (5) Article 7160.}

understanding of the social and biologic determinants of health has become readily apparent. Strategic curricular and innovative scholarly approaches for improving knowledge and developing competency in using evidence-based precision medicine tools among student pharmacists will bolster efforts to both advance the profession and contribute to reducing health disparities.

The Phrontier of Pharmacy: How Pharmacogenomics is Enhancing the Healthcare Field. Renee McCafferty, Manchester University, Teresa M. DeLellis, Manchester University, Diane M. Calinski, Manchester University, David F. Kisor, Manchester University, Thomas R. Smith, Manchester University, Carrie C. Hoefer, Manchester University. Pharmaceutical science programs incorporated pharmacogenomics (PGx) into research well before the Human Genome Project (HGP) was completed in 2003. The completion of the HGP not only enhanced PGx knowledge, but it was the start of incorporating precision medicine into healthcare. The 2016 ACPE standards require all pharmacy schools to implement PGx into the curriculum. Therefore, it is expected that all future pharmacists will not only be the drug-drug interaction experts, but also the drug-gene interaction experts. To integrate PGx into pharmacy, Manchester University College of Pharmacy, Natural \& Health Sciences (MUCPNHS) has implemented several curricular enhancements. For example, purposeful design and assessment of integrated pharmacotherapy courses offer unique opportunities to maximize the impact of PGx on student learning. Within these courses, patient cases involving polypharmacy and complex pharmacogenomic interactions are developed and presented by science and practice faculty. These PGx-informed patient cases are also leveraged to incorporate advanced precision medicine concepts into interprofessional education events. In this forum, PGx holds great promise for providing students with the framework to succeed in an interprofessional setting. Another avenue MUCPNHS uses to integrate PGx into Pharmacy is a lab-based elective. In this elective, pharmacy students generate PGx panel reports on themselves, reinforcing their PGx learning. Lastly, MUCPNHS now offers a dual degree program in which Pharmacy students can master the PGx curriculum, earning the nation's first dual PHRMD/MS in PGx degree. By offering this exclusive opportunity, MUCPNHS leads the way in ensuring future pharmacists will improve the healthcare field in precision medicine.

Threading Pharmacogenomics Education throughout the Core PharmD Curriculum: 4 Years of Experience Using Personal Genomic Testing. Philip E. Empey, University of Pittsburgh, Lucas A. Berenbrok, University of Pittsburgh, James M. Stevenson, University of Pittsburgh, James C. Coons, University of Pittsburgh, Solomon M. Adams, University of Pittsburgh. Objectives: Pharmacogenomics (PGx) education includes basic pharmaceutical sciences concepts and their application in pharmacy practice, but it is difficult to thread across core PharmD curricula. We report our highly successful integration of student personal genomic testing (PGT) across multiple courses to meet PGx curricular competencies and achieve high student engagement. Methods: Test2LearnTM is an educational model that integrates expert content, real genetic data, and structured learning activities within a responsible ethical framework using a custom web application. Learners may optionally undergo PGT (or use anonymous real genetic data) as a means to increase the fidelity of education. In 2014, we mapped PGx competencies to course objectives and curricular sequence and deployed Test2LeanTM in a year 2 pharmacokinetics course. Over the next 4 years, content was adapted for earlier, science-based courses and later, clinical applications courses. Feasibility, engagement, student attitudes, and learning outcomes were measured through participation metrics, objective assessments, and pre/post surveys. Results: 568 students participated over 4 years. New science- and application-based competencies were aligned with individual course objectives. The following concepts were covered: understanding the PGx basis of population variability, ethics, clinical trials, and regulatory guidance (Year 1); the science and implementation of PGx (Year 2); and finally PGx applications in pharmacy practice (Year 3). Engagement (PGT participation) was high (91.3\%; 519 of 568 students)]. Both subjective/objective knowledge assessments and PGx attitudes improved. Implications: Integration of PGT across the curriculum is feasible, highly engages students, and enables bridging of basic science and application to achieve competency-based learning outcomes.

Transparency + Structure $=$ Collaborations. Beth Ross, Pacific University Oregon, Pauline A. Low, Pacific University Oregon, Reza Karimi, Pacific University Oregon, Sarah Jane E. Faro, Pacific University Oregon, Ian C. Doyle, Pacific University Oregon, Anita J. Cleven, Pacific University Oregon, Rita R. Barton, Pacific University Oregon, Jeff Fortner, Pacific University Oregon. At Pacific University School of Pharmacy, our culture of administrative transparency and faculty driven governance, along with our non-departmental, flat organizational structure, encourage collaborations between pharmaceutical science (PS) and pharmacy practice (PP) faculty. These factors reduce competition and foster collegiality between PS/PP faculty and staff, resulting in the following accomplishments across various aspects of our program. Scholarship: PS/PP faculty collaborated on over two dozen papers, posters, and presentations since 2015. Many of these activities included students and staff, and contributed to the overall collegiality of our program. Teaching: PP support PS teaching via Clinical Correlates to bridge practice topics into science, SciencePractice-Connect activities integrate science into practice courses to promote application and critical-thinking skills, exam item writing groups include both PS/PP faculty, elective courses and learning tracks involve both PS/PP faculty, PS/PP collaborate to improve transitions of P1 students to P2 year. Service: Each School committee includes PS/PP faculty, our Office of Educational Research and Statistics is codirected by PP/PS faculty, practice faculty are invited to science conferences and meetings and vice versa, minutes from PS/PP meetings are disseminated to all faculty and staff so all cohorts are informed of updates. Awards: PS/PP collaborations resulted in two AACP Innovations in Teaching, and two Excellence in Assessment awards since 2010. Efforts to encourage transparency, and decisions to organize our program structure to increase collegiality, have resulted in productive collaborations between PS and PP faculty, with benefits for students, faculty, staff, and administration.

University of Mississippi School of Pharmacy "Phamily:" Collaborative Efforts of Faculty Development, Enrichment, and Engagement. David D. Allen, The University of Mississippi, Jason Paris, The University of Mississippi, Samir Anis Ross, The University of Mississippi, Narasimha Murthy, The University of Mississippi, Daniel M. Riche, The University of Mississippi, Yi Yang, The University of Mississippi, Kristopher Harrell, The University of Mississippi. Objective: To provide an overview of coordinated, collaborative efforts utilized to connect pharmaceutical science and pharmacy practice faculty. Faculty Development: The School hosts annually a Faculty and Research Scientist Retreat, intentionally alternating between Oxford and Jackson venues. These have dedicated time for practice faculty to visit research laboratories and science faculty to visit practice sites. They also typically include creative activities, many of which focus on enhancing teaching and research collaborations. Recent examples include Pharmentine's Day with Speed Dating and Beauty and the Beast 


\section{American Journal of Pharmaceutical Education 2018; 82 (5) Article 7160.}

with Coloring. Faculty Enrichment: The School sponsors a Brown Bag series focused on four research areas: cancer, cardiometabolic disorders, neuroscience/drugs of abuse, and infectious diseases. The monthly cross-campus discussions are held over the lunch hour (hence the term "Brown Bag") by videoconference. The aim is to provide an informal atmosphere for exchange of ideas. Additionally, the School hosts a more formal Research Day in which faculty, students, and postdoctoral fellows present posters and oral presentations, with the goal of promoting existing collaborations and facilitating additional opportunities. Faculty Engagement: As a direct result of retreats and implementation of a newly integrated curriculum, there is more crossdepartmental engagement. Practice and science faculty share teaching responsibilities in multiple courses. A research collaboration between the biomolecular sciences and practice faculty centered on antimicrobial stewardship was developed. Additionally, the pharmacy practice and pharmacy administration faculty launched a joint monthly meeting to promote scholarly faculty collaborations and the opportunity to learn from the skills and expertise existing amongst department faculty.

Use of Mapping to Assess Basic, Clinical, and Social Science Curricular Integration. Marc S. Abel, Rosalind Franklin University of Medicine and Science, Sarah S. Garber, Rosalind Franklin University of Medicine and Science, Scott D. Hanes, Rosalind Franklin University of Medicine and Science. Objective: Integration of basic, clinical, and social sciences within the pharmacy curriculum is recognized as a vital component of pharmacy education. The purpose of this study was to characterize basic, clinical, and social science integration within a PharmD curriculum without formally integrated courses. Methods: Courses were categorized as basic, clinical, or social science based. Course directors (CDs) completed a 4-question survey identifying basic, clinical, and social science content within their course as defined by ACPE Standards Appendix 1. Additionally, CDs were asked to characterize their level of interaction with other CDs regarding incorporation of content. Results: Mapping was completed for all courses. Clinical science courses incorporated $100 \%$ of all basic and social science content with pharmacology, pathophysiology, clinical chemistries, pharmaceutical calculations, pharmacokinetics, professional communications, and professional development being the most frequent cited. Basic science courses incorporated $70 \%$ and $73 \%$ of clinical and social science content, respectively, with medication dispensing, patient safety, clinical pharmacokinetics, pharmacotherapy, pharmacy history, and cultural awareness most frequently cited. Social science courses incorporated $50 \%$ and $12.5 \%$ of clinical and basic science content, respectively, with health informatics, patient safety, and public health being the most common cited. The majority of CDs indicated no or only limited purposeful coordination of content between CDs. Implications: Curricular mapping of the science disciplines identified curricular integration gaps and opportunities for optimization. Overall, science disciplines were well represented throughout the curriculum, however, several content areas may be underrepresented as integrated topics. Mechanisms to promote greater content coordination between disciplines and CDs are needed.

Using Simcyp Simulator and Principles in Clinical Pharmacology Course to Integrate Pharmaceutical and Clinical Sciences. Hieu T. Tran, California Northstate University, Chikere Nwokeji, California Northstate University, Vu Nguyen, California Northstate University, Suzanne Clark, California Northstate University, Ruth Vinall, California Northstate University, Rania Elkeeb, California Northstate University, Eman Atef, California Northstate University. California Northstate University College of Pharmacy (CNUCOP) has utilized several approaches to connect Pharmaceutical and Biological Sciences
(PBS) and Clinical and Administrative Sciences (CAS) faculty and students. The following are two newly used approaches: Two PBS faculty members are collaborating on a PK study to train PharmD students in Pharmacokinetics simulation projects. The aim is to help the students to integrate their basic science knowledge into clinical PK practice and health outcomes prediction. We use the Simcyp ${ }^{\circledR}$ Simulator, a physiologically based pharmacokinetics modeling and simulation platform that predicts drug disposition in multiple virtual human populations, including, but not limited to pediatrics, pregnant women and geriatrics. Simcyp ${ }^{\circledR}$ can be used to define dosage regimen, drugdrug interactions, and effect of food on absorption in an effort to predict clinical outcomes. Molecules of interest included furosemide and itraconazole with a PK focus on pediatric population. In addition, CNUCOP registered as a remote site for the on-line NIH Principles in Clinical Pharmacology course. Seven PBS and the CAS faculty are participating in this course. Participants meet monthly to discuss and reflect on course content in regards to its relation to our PharmD curriculum and research focus. The modules in the course include: Pharmacokinetics, Drug Metabolism and Transport, Pharmacokinetic and Drug Therapy in Special Populations, Assessment of Drug Effects, Drug Discovery and Development, Pharmacogenomics and Pharmacotherapy. The objectives of the course are to strengthen and improve the clinical and basic sciences knowledge of our faculty in both Departments and to promote future research collaboration.

Utilizing Case-Based Learning to Connect Science and Practice. Kristy L. Wahaib, Belmont University, Marilyn E. Thompson Odom, Belmont University. Within the pharmacy curriculum, basic science courses are the foundation upon which the clinical sciences build. To ensure a smooth progression between the two sciences, faculty must be knowledgeable of the content taught in each other's discipline. Despite adoption of integrated curricular models as encouraged by ACPE, foundational sciences are often not effectively utilized or appreciated by students as they progress in the curriculum and are encouraged to think critically and make therapeutic decisions. Direct demonstration of the connection between basic and clinical sciences could benefit student learning. To facilitate this, two faculty, one who teaches Pharmacodynamics and one who teaches Pathophysiology and Therapeutics, developed an integrated case-based lesson plan. Students in Pharmacodynamics III were assigned a case appropriate for a third semester student. After reading the case in class, students were asked to answer specific questions related to the basic sciences. In addition to concepts covered in Pharmacodynamics, including mechanism of action, indication, efficacy, adverse effects, and other therapeutic options, questions also covered cardiovascular anatomy and physiology, which was taught during the first semester of pharmacy school. Instructors were available to discuss the case with students after they completed the assignment. Following the session, feedback from students was favorable and supported continuation of the activity. Next year, the integrated case-based lesson plan will be implemented in the first year course, Anatomy \& Physiology, and expanded in years two and three in Pharmacodynamics III and Pathophysiology and Therapeutics III, respectively.

Utilizing Required PharmD Projects and Assistantships to Engage Students in Research across Disciplines. Aleda M. Chen, Cedarville University, Juanita A. Draime, Cedarville University, Melissa Beck, Cedarville University, Samson Amos, Cedarville University, Justin W. Cole, Cedarville University. Many areas of pharmacy practice require knowledge and application of research methodology. Incorporating opportunities to participate in research projects can assist PharmD students in developing skills and abilities in pharmacy practice 


\section{American Journal of Pharmaceutical Education 2018; 82 (5) Article 7160.}

(PP), social and administrative sciences (SAS), and pharmaceutical science (PS) research principles. At Cedarville University School of Pharmacy, students participate in required longitudinal research projects. In their first semester (P1 fall) research course, they rank projects offered by $\mathrm{PP} / \mathrm{SAS} / \mathrm{PS}$ faculty, are assigned to teams, and complete and present research proposals. From P1 spring to P3 fall, the students conduct their research projects. In the P3 spring, students take a research seminar course, during which they write a journal-style manuscript and present their results to faculty and students. Data from the first two cohorts indicate that these projects improved students' perceived understanding of the research process (7-point, Likert-type agreement, mean \pm SD: $5.98 \pm 0.72$ ). In addition, students are introduced to PP/SAS/PS research, including collaborative research between departments (23\% of projects). Students may also engage in extracurricular training programs offered by PP/SAS and PS departments and work on additional research projects to prepare for future fellowship and residency opportunities. For example, the PP/SAS department provides a semester-long, structured training series in scientific writing, data analysis, and methodology prior to working on research projects in a part- or full-time capacity. Students completing this training have engaged in post-graduate residencies and fellowships. Thus, a variety of research experiences can help students for future practice and improve understanding of how the $\mathrm{PP} / \mathrm{SAS} / \mathrm{PS}$ disciplines are related.

Working Together to Train PharmD Candidates in a Seminar Research Course. David Ross, University of Colorado, Molly M. Huntsman, University of Colorado, Robert MacLaren, University of Colorado, Kari L. Franson, University of Colorado. Introduction: PharmD-trained investigators make important contributions to therapeutics through research. The University of Colorado Skaggs School of Pharmacy \& Pharmaceutical Sciences (SSPPS) included a research component in its renewed curriculum. The vision was: "that all students develop and present a scholarly proposal in a manner that incorporates scientific principles of research design." As a result, SSPPS faculty facilitated groups of four students in a required two course series spanning the P2 Spring and P3 Fall semesters. Design: Faculty members from the Department of Pharmaceutical Sciences and Department of Clinical Pharmacy participate. Teams of students independently present a seminar on a faculty-assigned topic and derive a therapeutic question they wish to address using scientific principles. The P2 Spring semester focuses on the preparation and presentation of a research seminar with identification of a therapeutic question. The P3 Fall semester focuses on the preparation and presentation of a research poster on how to address the specific research problem the students have chosen. Students are evaluated by group products and individual effort. Results: Forty seminars and research posters have been presented each year since 2014. Topics average 1/3 from Pharmaceutical Sciences and 2/3 from Clinical Pharmacy. After participating, students have increasingly agreed with the AACP graduating survey question "I was aware of opportunities to participate in research activities with faculty." Conclusion: This two-part course series broadly exposes students to a variety of research. Students have demonstrated their research abilities and are increasingly aware of extracurricular opportunities to engage in research.

Work Together, Play Together: Interdepartmental Collaboration at WesternU. Anandi V. Law, Western University of Health Sciences, Stephen A. O'Barr, Western University of Health Sciences, Donald I. Hsu, Western University of Health Sciences. WesternU College of Pharmacy's (WesternU) Vision Statement is: "Develop leaders in pharmaceutical care and research who will advance global health outcomes through innovative pharmacy practice, interprofessional collaboration, scholarship and service." WesternU has long standing experience and leadership in interprofessional education, with a focus on interdisciplinary collaboration. The collaborative process begins when new faculty are given an opportunity to participate in a clinical practice site or lab visit as part of the new faculty orientation process and continues through interdepartmental interactions during teaching, scholarship and service activities. Teaching collaborations involve planning progression of didactic topics from pharmaceutical sciences to pharmacy practice, co-teaching, co-facilitation, and pedagogy and methods. Collaborative research occurs in many dyads of faculty and has resulted in combined grants, abstracts and peer reviewed publications in fields from SOTL to translational research. Service collaborations are numerous since committee members are appointed by both department chairs working together. Examples are faculty search committees which include a member of the other department and our Faculty Orientation and Development Committee, which has resulted in several collaborative publications. Finally, collaborative professional socialization abounds in the form of routine celebrations and informal lunches; and an annual College Ping Pong Tournament with plenty of camaraderie and a range of skills. A new development is an interdepartmental "Speed Dating" event which facilitates information exchange between faculty. The benefits from these collaborations are numerous and have been assessed. An environment of interdisciplinary collaboration and a culture of cooperation positively impact faculty engagement, recruitment, retention and satisfaction. 